\title{
Prototype hybrid couplings of macroscopic deterministic models and microscopic stochastic lattice dynamics
}

\author{
M. A. Katsoulakis, A. J. Majda, and A. Sopasakis
}

\begin{abstract}
We study a class of model prototype hybrid systems comprised of a microscopic Arrhenius surface process describing adsorption/desorption and/or surface diffusion of particles coupled to an ordinary differential equation displaying bifurcations triggered by the microscopic process. The models proposed here are caricatures of realistic systems arising in diverse applications ranging from surface processes and catalysis to atmospheric and oceanic sciences. Furthermore, we derive and study closures of this hybrid system by employing three different methods: deterministic closures through an averaging principle, mean field approximation and stochastic closures by employing a hierarchy of coarse grained models. We focus on analyzing the impact of microscopic fluctuations and interactions on the overall system's transient and long-time dynamics. For example fluctuation driven rare events may effect regimes exhibiting metastability. This type of phenomena can occur in several parameter regimes and typically they can not be accounted for by the deterministic closures. In contrast, the stochastic coarse grained closure gives rise to computationally inexpensive reduced hybrid models that capture correctly the behavior of the full microscopic system. This is further demonstrated in several Monte Carlo simulations testing a variety of parameter regimes and displaying numerically the extent, limitations and validity of the theory.
\end{abstract}

\section{Introduction}

A wide variety of physical applications ranging from catalysis, deposition processes and polymeric flows to complex biological networks and stochastic parameterizations for tropical and open ocean convection [MK02, VSA90, OL04, RJ98, WSL03] involve couplings of atomistic or molecular, and more generally microscopic stochastic models with deterministic macroscopic ordinary and partial differential equations (ODE/PDE). In this class of problems microscopic stochastic processes model small scale activity; for instance in catalysis such microscopic mechanisms can model adsorption, desorption, surface reaction and surface diffusion of particles on an interface or boundary layer which is in contact with a gas/fluid phase. The micro-mechanisms are thus interrelated with the large scale fluid flow

1991 Mathematics Subject Classification. 34E13; 82C27; 93C15; 37M20; 65C05;

Key words and phrases. Coupled Hybrid Systems; Stochastic Closures; Multi Scale Interactions; Critical Phenomena; Monte Carlo Methods;

The first author was supported in part by NSF-DMS-0413864, NSF-ITR-0219211 and DEFG02-05ER25702.

The second author was supported in part by NSF-CMG-0222133 and NSF-DMS-0456713. 
modeled by continuum ODE/PDE describing the evolution of fluid and thermodynamic variables. It is therefore not surprising that some of the major challenges which transcend these hybrid problems are related to disparities due to scale separation between the competing processes and a resulting computational inability for direct numerical simulations of realistic size systems. Furthermore, from a mathematical point of view, the influence of microscopic fluctuations and interactions on the overall behavior of the hybrid system could be significant, at least in some parameter regimes.

Here we focus on model prototype systems consisting of coupled macroscopic, deterministic differential equations and microscopic, stochastic lattice particle systems proposed in [KMS04] that are computationally tractable with direct numerical simulations even for long times, but still can exhibit a host of complex phenomena [KMS04, KMSb, Mur89]. Microscopic noise and interactions will indeed significantly alter the deterministic mean field behavior of the hybrid system yielding corresponding regimes with phenomena driven by the interaction of nonlinearity and noise across scales, such as strong intermittency, stochastic resonance, and random oscillations. Our findings strongly suggest [KMSb] that deterministic closures of the hybrid system such as mean field, stochastic averaging principle or moment equations may be either inadequate as descriptions of the overall system or simply difficult to both derive, as well as assess their effectiveness. In general fluctuations can play a dominant role in the stochastic system as is evident in long time simulations and asymptotic analysis in a linearized stochastic PDE limit. The influence of fluctuations is also pronounced in the case of short microscopic interactions where we demonstrated in $[\mathbf{K M S a}]$ that metastable oscillations and intermittency regimes may arise in the dynamics of the hybrid system. In general we show that fluctuation-driven rare events occur in several parameter regimes and are not captured by the deterministic mesoscopic equations. Along these lines, computational closure methods relying on relatively short runs of microsimulators such as the Equation-Free $\left[\mathbf{K G H}^{+} \mathbf{0 3}\right]$ and HMM [EE03] approaches may also have to account for such phenomena when deciding the integration time for the micro simulation and determining suitable coarse-grained observables such as a number of moments.

Motivated by such considerations, we proposed in [KMSb, KMSa] a class of stochastic closures based on systematic coarse-graining of the microscopic stochastic lattice dynamics that yield computationally inexpensive reduced hybrid models and capture correctly the transient and long-time behavior of the full hybrid system. The proposed models describe the microscopic mechanisms with a hierarchy of coarser but still discrete, stochastic observables while maintaining a controlled error in the approximation. Furthermore the models allow for testing the derivation of computationally inexpensive mesoscopic deterministic and stochastic models for the average behavior of the hybrid systems in various asymptotic limits.

The outline of this paper is as follows: we introduce a system of microscopic stochastic surface processes in Section 2 modeling adsorption/desorption and/or surface diffusion of particles with spin flip/exchange Ising systems, coupled to an ODE that serves as a caricature of the gas-phase, exhibiting a similar variety of dynamic and stability behaviors in different parameter regimes. Examples of such ODEs are scalar equations exhibiting bistability and saddle node bifurcations, as 
well as a spatially homogeneous complex Ginzburg-Landau equation exhibiting a Hopf bifurcation behavior.

Our work on these problems addresses two main themes. First in Section 3.1 we derive deterministic mesoscopic, spatially distributed equations from the microscopic hybrid system by assuming long range interactions on the microscopic processes. Then in Section 3.3 we derive deterministic mesoscopic models by employing a stochastic averaging principle (see for instance [FW98] and references therein) to the microscopic lattice model. The stochastic averaging closure is carried out rigorously under the assumption that the stochastic mechanism is faster than the gas-phase (ODE) relaxation, inducing a local equilibrium with respect to the Gibbs measure on the lattice system. In both asymptotic regimes the resulting averaged equations can be thought as deterministic coarse-grained models providing some first intuition on the interplay of various mechanisms.

All theoretical results are derived on finite-time intervals, where the influence of noise asymptotically vanishes due to the Law of Large Numbers scalings that are typically employed. We refer to the relevant discussion and study in [KMS04] where extensive comparative simulations with both the hybrid system and the mesoscopic deterministic closures display agreement in the cases for which the assumptions regulating the derivations are not violated. However simulations for very long times with our hybrid prototype models, as well as linearization arguments, strongly suggest that fluctuation-driven rare events do occur in several parameter regimes and are not captured by the deterministic averaged equations discussed above. This is hardly surprising in view of the results in [FW98] on large deviations for averaged systems. Furthermore, random fluctuations are especially important in phase transition regimes even in an uncoupled lattice model. As a result it is clear that fluctuations need to be properly included in any coarse-grained mesoscopic model. We address this issue in Section 5 by employing recently developed coarse-grained stochastic models [KMV03a, KMV03b, KV03], in order to describe the microscopic mechanisms with a hierarchy of coarser stochastic observables. We follow up in Section 7 with several numerical test cases validating the coarse grained stochastic closure for several different parameter regimes. For comparison purposes we also display results involving both uncoupled and fully coupled systems.

In Section 8 we study the intrinsically more complex case where randomness, macroscopic dynamics, as well as microscopic interactions compete and may dramatically affect the hybrid system behavior. Specifically, we examine the influence of phase transitions and metastability in the noisy micro-/sub- grid scale on the overall dynamics in the context of our simple prototype hybrid system. The examples treated here are dominated by strong interactions between scales and fluctuations and can demonstrate nucleation, intermittency and random oscillations. Furthermore a particularly simple example of these subtle behaviors is demonstrated in Subsection 8.1, where we consider a hybrid system with uniform, Curie-Weiss type interactions in the microscopic lattice dynamics; the uncoupled Curie-Weiss spin model is an important example in statistical physics that exhibits phase transitions, hysteresis, critical fluctuations and other related phenomena and allows for fairly simple yet revealing analytical calculations, [Ell85, LB99]. In the presence of strong particle/particle interactions in the lattice system compared to the random fluctuations, the mean field approximation of the proposed hybrid system gives an initial insight on the overall dynamics: the mean field system applied for these 
examples is a FitzHugh-Nagumo-type ODE that exhibits in different parameter regimes, excitable, oscillatory and bistable behaviors. Therefore, it is not surprising that the presence of noise will substantially modify these deterministic mean field FitzHugh-Nagumo behaviors and yield corresponding regimes with phenomena driven by the interaction of nonlinearity and noise across scales, such as strong intermittency, stochastic resonance, and random oscillations.

\section{A prototype hybrid model}

We introduce the microscopic spin flip stochastic Ising process $\left\{\sigma_{t}\right\}_{t \geq 0}$, modeling the adsorption and desorption of particles on a one-dimensional surface, coupled to an ODE that serves as a caricature of an overlying gas-phase dynamics; we also refer to [VSA90] for such a system arising in the modeling of well-mixed reactors in catalysis.

The microscopic stochastic process is defined by its' generator $L$ and the twoway coupled system is written as

$$
\begin{aligned}
\frac{d}{d t} \vec{X} & =\frac{1}{\tau_{c}} G(\vec{X}, \bar{\sigma}) \\
\frac{d}{d t} E f(\sigma) & =E L f(\sigma)
\end{aligned}
$$

where $\bar{\sigma}$ denotes a spatial average coverage, $f$ is a test function, $G$ the ODE function and $\tau_{c}$ corresponds to the characteristic time for the ODE. We provide all further details for system $(2.1,2.2)$ below. Examples of ODEs such as (2.1) considered here are scalar equations with bistable behavior or saddle node bifurcations, as well as a spatially homogeneous complex Ginsburg-Landau equation exhibiting Hopf bifurcations. In addition to the spin flip mechanism, we can also consider spin exchange, as well as combined spin flip / spin exchange mechanisms. In this paper we concentrate only on one of them, namely spin flips.

2.1. Microscopic Arrhenius dynamics. We start with a detailed description of (2.2). We consider a microscopic stochastic model defined on a periodic lattice of size $N$ which we denote by $\mathcal{L}=\{1,2, \ldots, N\}$. At each lattice site $x \in \mathcal{L}$, an order parameter $\sigma$, is allowed to take the values 0 or 1 . In accordance with the classical Ising model we refer to the order parameter as spin. We will assume that sites cannot be occupied by more than one particle. A spin configuration $\sigma$ is an element of the configuration space $\mathcal{H}_{N, 1}=\{0,1\}^{\mathcal{L}}$ and we write $\sigma=\{\sigma(x): x \in \mathcal{L}\}$ denoting by $\sigma(x)$ the spin at $x$. Physically this mechanism may describe the desorption of a particle from a surface described by the lattice to the gas phase above and conversely the adsorption of a particle from the gas phase to the surface. Similarly it can describe phase transitions without order parameter conservation although here we concentrate on the case where phase transitions are absent in the microscopic system.

The stochastic process $\left\{\sigma_{t}\right\}_{t \geq 0}$ is a continuous time jump Markov process on $L^{\infty}\left(\mathcal{H}_{N, 1}, R\right)$ with generator, [KL99],

$$
L f(\sigma)=\sum_{x \in \mathcal{L}} c(x, \sigma)\left[f\left(\sigma^{x}\right)-f(\sigma)\right]
$$


for any test function $f \in L^{\infty}\left(\mathcal{H}_{N, 1}, R\right)$. Here $\sigma^{x}$ signifies the configuration after a flip at $x$,

$$
\sigma^{x}(y)=\left\{\begin{array}{cc}
1-\sigma(x), & \text { if } y=x \\
\sigma(y), & \text { if } y \neq x
\end{array}\right.
$$

and $c(x, \sigma)$ denotes the rate of a spin flip at $x$ for the configuration $\sigma$ (see 2.6).

Since there are $N=|\mathcal{L}|$ sites on the lattice then the system can be in any of $2^{|\mathcal{L}|}$ possible states while the energy of any particular state is given by the following Hamiltonian,

$$
H(\sigma)=-\frac{1}{2} \sum_{x \in \mathcal{L}} \sum_{y \neq x} J(x, y) \sigma(x) \sigma(y)+\sum_{x} h \sigma(x) .
$$

where $J(x, y)$ is an inter-particle potential and $h$ is a given external potential. We let,

$$
J(x, y)=\frac{1}{2 L+1} J\left(\frac{|x-y|}{2 L+1}\right)
$$

where $L$ denotes the range of microscopic interactions. Here $J$ is assumed to be even, $J(r)=J(-r)$ and as an example, can take a form similar to [KMV03a],

$$
J(r)= \begin{cases}J_{0} & \text { if } 0 \leq r \leq 1 \\ 0 & \text { otherwise }\end{cases}
$$

where $J_{0}$ is a parameter which based on its sign describes attractive, repulsive or no-interactions. For attractive microscopic interactions for instance, $J_{0}$ is positive.

By applying a spin flip stochastic model we create new states from old ones generated by a Markov process as described below. Equilibrium states of the stochastic model are described by the Gibbs states at the prescribed temperature $T$. If we denote the inverse temperature by $\beta=1 /(k T)$ we then have,

$$
\mu_{\beta, N}(d \sigma)=\frac{1}{Z} e^{-\beta H(\sigma)} P_{N}(d \sigma)
$$

where $P_{N}(d \sigma)$ denotes the (product) prior distribution on $\mathcal{L}$,

$$
P_{N}(d \sigma)=\prod_{x \in \mathcal{L}} \rho(d \sigma(x)) \text { and } \rho(\sigma(x)=0)=\frac{1}{2}, \quad \rho(\sigma(x)=1)=\frac{1}{2} .
$$

Here $Z$ is the partition function, guaranteeing that (2.5) is a probability measure.

In this model we implement spin flip Arrhenius dynamics. Under this type of mechanism the simulation is driven based on the energy barrier a particle has to overcome in flipping from one state to another given be (2.7) below. For Metropolis and other choices of dynamics see [KMV03a]. The Arrhenius spin flip rate $c(x, \sigma)$ at lattice site $x$ and spin configuration $\sigma$ is given by

$$
c(x, \sigma)= \begin{cases}c_{d} e^{-\beta\left[U_{0}+U(x)\right]}, & \text { when } \sigma(x)=1, \\ c_{a} & \text { when } \sigma(x)=0 .\end{cases}
$$

where,

$$
U(x)=\sum_{\substack{z \neq x \\ z \in \mathcal{L}}} J(x, z) \sigma(z)-h .
$$

with absorption / desorption constants, $c_{a}=c_{d}=1 / \tau_{I}$ and $\tau_{I}$ denotes the characteristic time of the stochastic process. Here $U_{0}$ represents the energy associated with the surface binding of the particle at location $x$ which we set to $U_{0}=0$. 
In general we write the spin flip rate (2.6) as,

$$
c(x, \sigma):=c_{a}(x, \sigma)+c_{d}(x, \sigma)=c_{a}(1-\sigma(x))+c_{d} \sigma(x) \exp \left(-\beta\left(U_{0}+U(x)\right)\right.
$$

and therefore the probability of a spin flip at $x$ during time $[t, t+\Delta t]$ is,

$$
c(x, \sigma) \Delta t+O\left(\Delta t^{2}\right) .
$$

The dynamics as described here leave the Gibbs measure (2.5) invariant, since they satisfy the detailed balance condition

$$
c(x, \sigma)=c\left(x, \sigma^{x}\right) \exp \left(-\beta \Delta_{x} H(\sigma)\right)
$$

where

$$
\Delta_{x} H(\sigma)=H\left(\sigma^{x}\right)-H(\sigma) .
$$

Similar combined mechanisms coupled with (6.1) were considered in [VSA90]. In this paper we focus for simplicity on the spin flip case only.

In the case of a surface diffusion process we implement spin-exchange Arrhenius dynamics. A spin exchange between the neighboring sites $x$ and $y$ is a spontaneous exchange of the values of the order parameter $x$ and $y$. Physically this mechanism describes the diffusion of a particle on a flat surface. Similar combined mechanisms were considered in [VSA90]. In this paper we focus for simplicity on the spin flip case only.

2.2. Couplings with deterministic systems. We present below some of the candidate examples for our ODE (2.1). The formulations studied were chosen in order to display interesting solution profiles through succinct and simple equations. They include linear, bistable, saddle and Complex Ginzburg Landau (CGL) equations [AK02]. We summarize here the main types of examples and stability behavior which we will study in more detail later. All parameters involved in the formulations below are explicitly provided in the simulation Section 7.2.

- Example 1 (Bistable):

$$
G(X, \sigma)=a(\bar{\sigma}) X+\tilde{\gamma} X^{3}, \quad \text { where } \quad a(\bar{\sigma})=b(1-\bar{\sigma})+z \quad \tilde{\gamma}<0
$$

In particular bistability arises according to the sign of $a(\bar{\sigma})$. If $a(\bar{\sigma}) \leq 0$ we have a stable node at 0 while otherwise we have two stable nodes at $\pm \sqrt{-a(\bar{\sigma}) / \tilde{\gamma}}$.

- Example 2 (Saddle):

$$
G(X, \sigma)=a(\bar{\sigma})+\tilde{\gamma} X^{2}, \quad \text { where } a(\bar{\sigma})=b(z-\bar{\sigma})
$$

Similarly this example displays saddle behavior depending on the sign of $a(\bar{\sigma}) / \tilde{\gamma}$. For $a(\bar{\sigma}) / \tilde{\gamma}>0$ there exist no nodes while otherwise we obtain one stable and one unstable node at $\pm \sqrt{-a(\bar{\sigma}) / \tilde{\gamma}}$.

- Example 3 (CGL):

$$
G(\vec{X}, \sigma)=\left[\left(\begin{array}{cc}
a(\bar{\sigma})+\gamma & -\omega \\
\omega & a(\bar{\sigma})-\gamma
\end{array}\right)-\tilde{\gamma}|\vec{X}|^{2}\right] \vec{X}
$$

where $a(\bar{\sigma})=b(\bar{\sigma}-z)$ and $\vec{X}=X+Y i$. This example exhibits a Hopf bifurcation. The Jacobian of the linearized system has eigenvalues $\lambda=\lambda(\bar{\sigma})=$ $a(\bar{\sigma}) \pm i \sqrt{\omega^{2}-\gamma^{2}}$ and in general we have either a stable node at $(0,0)$ for $a(\bar{\sigma})<0$ or a limit cycle.

- Example 4 (Linear):

$$
G(X, \sigma)=\omega \bar{\sigma}+b-c X .
$$


The ODE (2.1) and the stochastic system (2.2) are coupled via the external field and the area fraction (or total coverage) respectively,

$$
h \equiv h(\vec{X}), \quad \bar{\sigma}=\frac{1}{N} \sum_{x \in \mathcal{L}} \sigma(x) .
$$

The parameters in each example are chosen so that the noise constantly probes the critical values for which bifurcations occur under each of the examples above. The coupled system therefore can move between regions of stability or instability based on the coupling noise parameter $\bar{\sigma}$. Further details on the exact values of all parameters used and respective behavior of the bifurcations are to be found in the numerical simulations Section 7.2.

Each of the bifurcation ODEs $(2.10$ - 2.13) that enters in system $(2.1,2.2)$ has its own time scale, $\tau_{c}$ and competes with the characteristic time for the stochastic model $\tau_{I}$. We define $\tau=\tau_{c} / \tau_{I}$ and note the following three possibilities:

- $\tau>>1 \Longrightarrow$ ODE equilibrates slower than spin flip model.

- $\tau \approx 1 \Longrightarrow$ ODE equilibrates at similar times as the spin flip model.

- $\tau<<1 \Longrightarrow$ ODE equilibrates faster than spin flip model.

where without loss of generality we fix $\tau_{I} \equiv 1$ and vary $\tau_{c}$ accordingly for the cases specified above.

We will monitor in our simulations how the phase portrait of our dynamical system $(2.1,2.2)$ transforms when the critical parameter coupled to $\bar{\sigma}$ passes through its bifurcating value. We perform these numerical comparisons under the three cases of $\tau_{c}$ outlined above and a variety of values for the fixed parameters of the external potential and the ODEs corresponding to a wealth of different phase space portraits.

\section{Deterministic Closures and the Role of Stochastic Fluctuations}

In this section we outline deterministic closures of the hybrid system (2.2), (2.1) studied in [KMS04] in two distinct asymptotic regimes. First, in the case of weak long range interactions $L \rightarrow \infty$, we obtain a coupled, possibly spatially distributed mean field deterministic mesoscopic system for the evolution of $X$ and the average local coverage corresponding to the stochastic order parameter $\sigma$. Second, using stochastic averaging, we obtain an effective ODE for the variable $X=X(t)$ in the time-asymptotic limit $\tau>>1$, i.e. when the relaxation time $\tau_{I}$ of the stochastic system (2.2) is much faster than the relaxation time $\tau_{c}$ of the ODE (2.1). The rigorous derivations for these closures can be found in [KMS04].

3.1. Local and Global Mean Field Models. In this subsection we obtain in the asymptotic limit of long range interactions $N=2 L+1$ for $N, L \rightarrow \infty$ a spatially distributed deterministic closure of (2.2), (2.1), without any time-scales separation assumption. In that case, equilibrium mean-field theory becomes asymptotically exact (see [KMS04]). We briefly outline here the derivation of mean-field equations for our coupled system (2.2), (2.1). We consider the spin flip Arrhenius type dynamics which we presented in Section 2, and using the definition of the generator, we obtain the following time evolution law for the average coverage $E \sigma(x)$ and the average position $E \vec{X}$ :

$$
\left\{\begin{aligned}
\frac{d}{d t} E \vec{X} & =\frac{1}{\tau_{c}} E G(\vec{X}, \bar{\sigma}) \\
\frac{d}{d t} E \sigma(x) & =\frac{1}{\tau_{I}} E\left[(1-\sigma(x))-\sigma(x) e^{-\beta U(x ; \vec{X}, \sigma)}\right]
\end{aligned}\right.
$$


where $U(x ; \vec{X}, \sigma)=\sum_{z \neq x} J(x, z) \sigma(z)-h(\vec{X})$. Note that (3.1) is exact but not closed in terms of $E \sigma(x)$ and $E X$. Although such a system cannot provide a predictive tool by itself, it can certainly be used, since it is exact, as a numerical benchmark for the deterministic and stochastic closures presented next.

In order to proceed further and close the system above we consider an averaged but still stochastic quantity, namely the empirical measure,

$$
\mu^{N}(d y, t)=\frac{1}{N} \sum_{x \in \mathcal{L}} \sigma_{t}(x) \delta_{x}(d y), \quad \text { for } \quad y \in R
$$

Note that if $A \subset R$ then

$$
\mu^{N}(A, t)=\frac{\text { number of particles in } A}{N} .
$$

In this case our ODE, (2.1) becomes exactly,

$$
\frac{d \vec{X}}{d t}=G\left(\vec{X}, \bar{\mu}^{N}\right) \quad \text { with } \quad \bar{\mu}^{N}(t)=\int_{R} \mu^{N}(d z, t)=\bar{\sigma}_{t} .
$$

Passing to the weak-* limit in $\mu^{N}(d y, t)$ we have the following result: for a smooth long-ranged potential $J(x, y)=\frac{1}{N} J\left(\frac{|x-y|}{N}\right)$ and any finite time $T$, we have that

$$
\lim _{N \rightarrow \infty} X(t)=Y(t) \quad \text { and } \quad \lim _{N \rightarrow \infty} \mu^{N}(d y, t)=u(y, t) d y \quad \text { uniformly in }[0, T]
$$

where for $t \in[0, T]$ and $y \in[0,1],(Y, u)$ solves

$$
\left\{\begin{aligned}
\frac{d}{d t} \vec{Y} & =\frac{1}{\tau_{c}} G(\vec{Y}, \bar{u}) \\
\frac{d}{d t} u & =\frac{1}{\tau_{I}}\left\{1-u-u e^{-\beta(J * u-h(\vec{Y}))}\right\}
\end{aligned}\right.
$$

where $\bar{u}(t)=\int_{0}^{1} u(y, t) d y$ and $G$ corresponds to the given ODE as in (2.10), (2.11), (2.12) or (2.13). As discussed in [KMS04] relation (3.5) is analogous to the Law of Large Numbers yielding the mean behavior of the hybrid system and treating fluctuations as a higher order correction.

In all of the cases outlined above, the hybrid stochastic system may display transient and long-time behavior which can be completely different from those predicted by the deterministic closures, hence incorporating stochasticity in any closure scheme is an essential task. We therefore propose in the following section a stochastic closure which as we will show with numerical simulations in Sections 8.1 and 7 can alleviate these problems by accurately approximating our microscopic hybrid solutions. As a special case of coarse graining we will examine an exact coarse graining, the Curie-Weiss limit, in Section 8.1. This will further allow us to test our deterministic closures against the microscopic hybrid system.

A particular but important sub-case follows here if we make the assumption that $J \equiv J_{0}$ i.e assuming a uniform interaction potential. We subsequently obtain the following mean field model ODE system describing total spatial averages,

$$
\text { Mean-field: }\left\{\begin{array}{l}
\frac{d}{d t} \vec{Y}=\frac{1}{\tau_{c}} G(\vec{Y}, \bar{u}) \\
\frac{d \bar{u}}{d t}=\frac{1}{\tau_{I}} F(\vec{Y}, \bar{u})
\end{array} \quad \text { for } \quad t \in[0, T]\right.
$$

where $F(\vec{Y}, \bar{u}) \equiv(1-\bar{u})-\bar{u} e^{-\beta J_{0} \bar{u}+\beta h(\vec{Y})}$. The exact mean-field formulations corresponding to each of our examples will be presented in the simulations Section 7.2 below. We note here that the (uncoupled) Curie-Weiss lattice spin model of (2.2) is a classic simple example in statistical mechanics that exhibits phase transitions 
even in one space dimension and allows for detailed explicit calculations such as mean field and thermodynamic limits at all temperatures. As further detailed in Section 5, the Curie-Weiss hybrid system analogue can provide some first insights in a simple context on the influence of noise and phase transitions on the overall hybrid system dynamics by comparing it against the mean field closure (3.7). In fact, this example underlines some of the shortcomings of deterministic closures and motivates the stochastic closures in the following section that can allow us to overcome the related issues.

3.2. The role of phase transitions. We present in more detail the behavior of the mean field closure (3.7) with respect to different values of $\beta J_{0}$. The phase transition regime for the microscopic lattice system which appears for $\beta J_{0}>4$ can be seen in Figure 1.

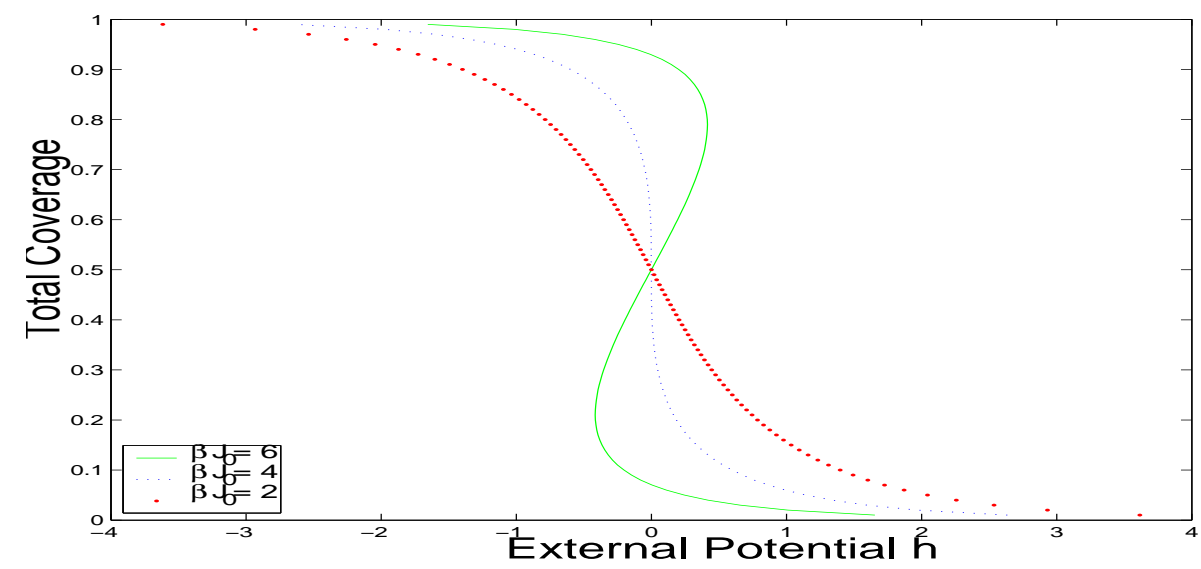

FiguRE 1. The total coverage for the uncoupled $\bar{u}$ from [KMS04] for different values of $\beta J_{0}=2,4$ and 6 versus fixed, not depending on $X, h \equiv h(X)$ external potential. The critical point for the $\{0,1\}$ spins satisfies $\beta_{c} J_{0}=4$. The hysteresis shape of the curve for the phase transition regime, $\beta J_{0}=6$, manifests existence of two equilibria in the neighborhood of the zero external field.

For simplicity we focus on the phase portrait of the mean field system (3.7) with linear $G$ (2.13) for the cases of no phase transitions $\beta J_{0}=2$ and phase transitions $\beta J_{0}=6$ is shown in Figure 2. Note that in the non-phase transition regime $\beta J_{0}<\beta_{c} J_{0}=4$, shown in Figure 2a, the zero level curve of the bottom equation in (3.7) becomes monotone and can only produce a single excitable state. This is in sharp contrast to the much richer behavior which the mean field system (3.7) displays in the remaining three subplots of Figure 2. We observe three types of stability profiles:
(a) excitable,
(b) bistable,
(c) oscillatory

depending on the parameters chosen for our mean field system in this phase transition regime $\beta J_{0}=6$ in Figures $2 \mathrm{bcd}$. As a result we emphasize that the behavior displayed in the numerical simulations which follow is attributed to the phase transition regime chosen. 

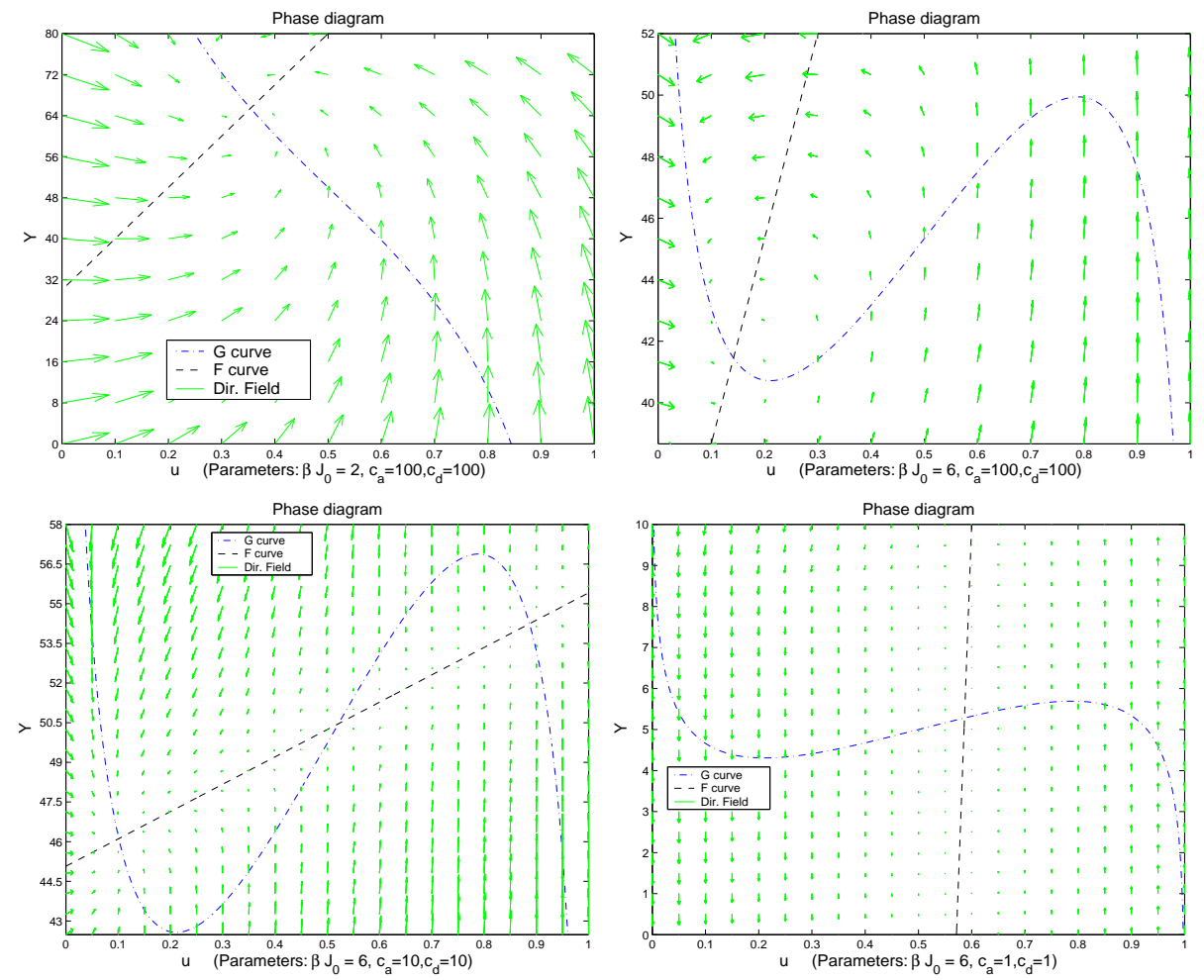

FiguRE 2. Direction field and phase portrait for mean field system (3.7) under different parameter regimes. Cases of excitability for $\beta J_{0}=2$ and excitability, bistability and oscillations for $\beta J_{0}=6$ respectively. Compare with pg. 328-329 of [Mur89]. The behavior shown here is exactly the same for the FitzHugh-Nagumo equation shown in (3.8).

A catalysis system which is similar to (3.7), was first studied in [KSA84] predicting oscillations both in the coverage and the pressure but it disregarded fluctuations, detailed interactions and spatial inhomogeneities. For this catalysis system, comparisons between mean field and Monte Carlo simulations [VSA90] showed that in many parameter regimes there is substantial agreement attributed to the coupling with the well-mixed gas-phase; drastic discrepancies were also observed, as well as a variety of dynamic and equilibrium behaviors, depending on the separation of time scales between individual micro-mechanisms and the time scale of the ODE.

The importance of phase transitions in a coupled system can be seen immediately, in the simple case of (2.13), by noticing that (3.7) has the same behavior as a typical FitzHugh Nagumo ODE,

$$
\frac{d}{d t} x=\omega u+b-c x, \quad \text { and } \quad \frac{d}{d t} u=g(u)-x
$$

where usually $g(u)$ is nonlinear $g(u)=u(a-u)(u-1)$ as in [Mur89] (pg. 329). System (3.8) has been extensively examined in several publications since, although 
simple in form, it is capable of exhibiting interesting behavior in different parameter regimes. In fact, system (3.8), displays exactly the same phase profile as (3.7) exhibits in Figure 2 for $\beta J_{0}=6$ with linear $G$ (2.13) (see also Figs. 6.8, and 12.6 in [Mur89]).

3.3. Stochastic Averaging. We now assume that $\tau>>1$ which implies that the stochastic system evolves with a characteristic time which is much faster compared to that of the ODE. In this case we can apply a stochastic averaging principle, which is conceptually similar to the one for systems of stochastic differential equations with fast and slow scales, see for instance [FW98].

The main requirement here is the ergodicity property of the stochastic process $\left\{\sigma_{t}\right\}_{t \geq 0}$. Indeed, for any fixed, finite $N$ and $\vec{X}$, the process $\sigma$ is ergodic with (2.5) as the unique invariant measure, [Lig99]; in particular we have,

$$
\lim _{T \rightarrow \infty} \frac{1}{T} \int_{0}^{T} G\left(\vec{X}, \overline{\sigma_{t}}\right) d s=\bar{G}(\vec{X}) \text { for } \quad \vec{X} \in R^{2}
$$

where

$$
\bar{G}(\vec{X})=E_{\mu_{\beta, N}} G(\vec{X}, \bar{\sigma}) \text { for } \quad \vec{X} \in R^{2}
$$

Due to special structure of $G$ which depends on $\bar{\sigma}$ linearly (see $(2.10,2.11,2.12)$ ), we always have

$$
E_{\mu_{\beta, N}} G(\vec{X}, \bar{\sigma})=G\left(\vec{X}, u_{\beta, N}(h(\vec{X}))\right)
$$

where,

$$
u_{\beta, N}(h)=E_{\mu_{\beta, N}} \bar{\sigma}=\frac{1}{Z} \sum_{\{\sigma\}} \sum_{x \in \mathcal{L}} \sigma(x) e^{-\beta H(\sigma)} P_{N}(\sigma) .
$$

Furthermore (3.11) is the average coverage of the microscopic system which in equilibrium statistical mechanics is known as average magnetization. It is clear from the formula that for finite $N, u_{\beta, N}(h)$ is an analytic function of $h$, we refer to [Ell85] (Theorem V.4.3) for a detailed discussion of its properties (e.g. monotonically increasing, concave for $h>0$, symmetry in $h$, etc.). In other words there are no first-order phase transitions for finite $N$, and in this case (3.10) and the averaged ODE (3.13) below are always well defined, regardless of temperature and strength of interactions. In fact we can show the following: for an arbitrary bounded time interval $[0, T]$ with fixed $N$ and $\tau_{c}$ we have

$$
\lim _{\tau_{I} \rightarrow 0} P\left(\sup _{0 \leq t \leq T}|\vec{X}(t)-\bar{x}(t)|>\delta\right)=0,
$$

for any $\delta>0$ where $\vec{X}=\vec{X}(t)$ solves (2.1), and $\bar{x}=\bar{x}(t)$ is the solution of the averaged system (depending on the lattice size $N$ )

$$
\begin{cases}\frac{d}{d t} \bar{x}_{t} & =\frac{1}{\tau_{c}} \bar{G}\left(\bar{x}_{t}\right) \\ \bar{G}\left(\bar{x}_{t}\right) & =G\left(\bar{x}_{t}, u_{\beta, N}\left(h\left(\bar{x}_{t}\right)\right)\right) \quad \text { for } t \in[0, T] . \\ \bar{x}_{0} & =x\end{cases}
$$

Remark 3.1. For a finite $N$, the spin flip system has no phase transitions which can arise depending on the competition between fluctuations and interactions, in the $N \rightarrow \infty$ limit. Therefore the results above always apply since in that context $N$ is kept fixed, while $\tau_{I} \rightarrow 0\left(\tau=\tau_{c} / \tau_{I} \rightarrow \infty\right)$. However, even for finite $N$, phase transitions are manifested as hysteresis in Monte Carlo simulations (see for instance Figures 1 and 2 in [KMV03a]). A similar phenomenon arises also 
here, since the coupling of (2.1) to the spin flip system is through the external field $h=h(\vec{X})$. The phase transition case in the stochastic lattice system will be examined extensively in [KMSa]; in this case the coupled system $(2.1,2.2)$ not only demonstrates metastability but also oscillations and intermittency.

For issues related to numerical implementation of the averaged system (3.13) and in particular the calculation of $u_{\beta, N}(h)$ we refer to [KMS04]. Once $u_{\beta, N}(h)$ has been determined it is an easy task to obtain the corresponding averaging closure for each of our ODE examples. In general, based on all examples presented in [KMS04] (and several others not reported there) the average principle predictions and the Monte Carlo simulations completely agree for the parameter regimes for which this theory holds (i.e. faster stochastic $\tau_{I}<<\tau_{c} \rightarrow \infty$ ).

\section{Validity Regimes of Deterministic Closures}

Both of the closures presented in the previous section have been successful under extensive numerical tests in [KMS04] when applied reasonably within the limits of their respective underlying assumptions as outlined in the previous section. In this section we examine the impact of applying these closures in situations for which the underlying mathematical assumptions are expected to be violated and noise could be important.

More specifically one important underlying assumption for both the stochastic averaging and mean field closures is that they are valid for a finite time interval $[0, T],[$ KMS04]. In the case of infinite time intervals, even for small noise, large deviation effects may be present which can alter the stability of the hybrid system. Similarly if the coupling is subjected to high noise levels, (e.g. $\tau<<1$ ) deterministic closures are expected to fail. Alternatively changes in the system's temperature or in strength of interactions (see Fig. 2) can create phase transitions, which in turn can generate a whole new set of phenomena, such as intermittency or oscillations, which do not exist in the uncoupled spin flip system. In all of the cases outlined above, the hybrid system may display transient and long-time behavior which can be completely different from those predicted by the deterministic closures. Chosen examples presented in this section display the capabilities as well as the shortcomings of deterministic closures. For instance, a case where stochastic fluctuations may be critical is the blow-up (solutions tending to $\infty$ ) examples which are studied in Section 4.2. In this case the analogue of (3.13) cannot predict blow-up which occurs for the direct numerical simulations of the full stochastic model.

Before we start examining the limitations for the deterministic closures presented in Section 3 we should briefly present a characteristic case where the approximation works well within the limits of validity of the underlying assumptions. As a typical test case of success for the stochastic averaging closure (3.13) we present a solution of our coupled system $(2.1,2.2)$ for the Hopf bifurcation ODE (2.12) in Figure 3. We solve our hybrid model under the case of $\beta J_{0}=2$ and $\tau=1$. Note that here we are away from the phase transition regime which occurs for $\beta J_{0}=4$. Although the case $\tau=1$ is not $>>1$ as required by the mathematical theory (3.12), this stochastic averaging type closure still performs well and approximates the solutions for coupled systems with fixed stable equilibria or single periodic orbit as long as we stay within the assumptions of its derivation. Several more such examples have been undertaken under different types of bifurcation couplings and a small fraction have been presented in [KMS04]. 

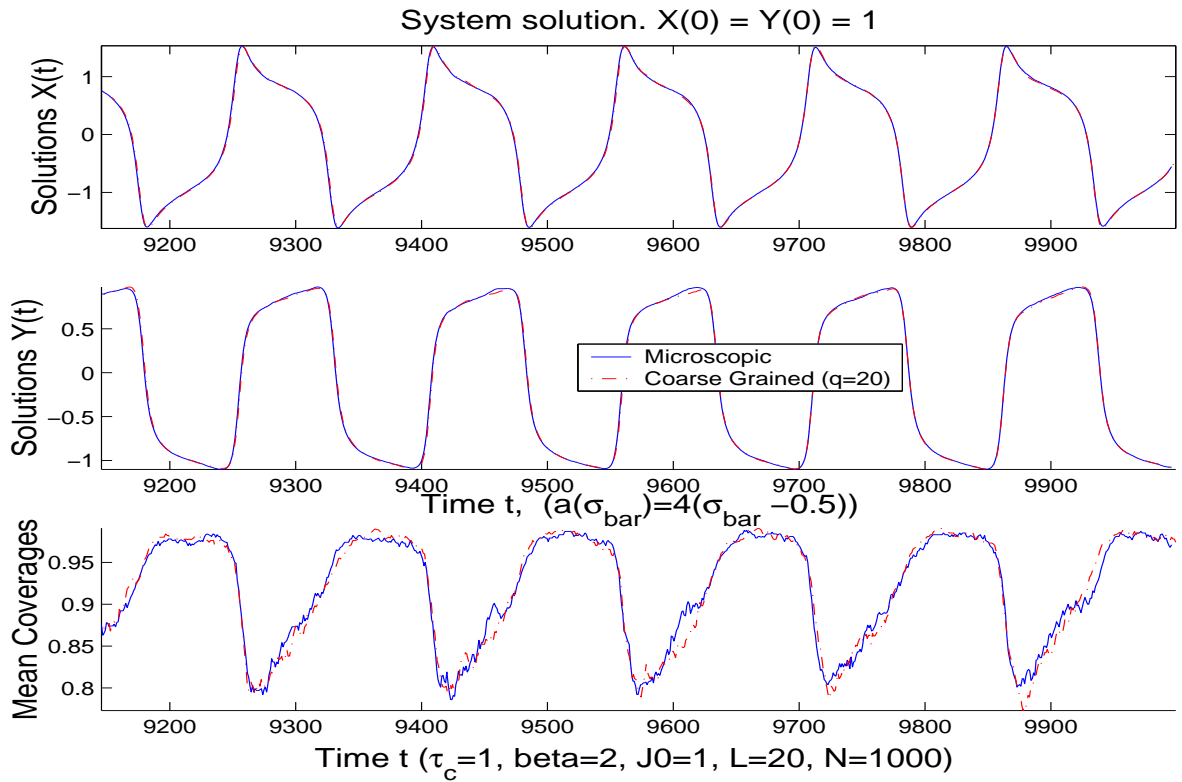

Figure 3. Typical example of the stochastic averaging closure producing the correct solution. Comparisons of solutions in time for the microscopic coupled system versus the stochastic averaging closure. See [KMS04] for a discussion and details of the solutions. Parameters $\tau=1$ and $\beta J_{0}=2$.

We next examine four examples where stochastic fluctuations will be important. Under these circumstances we will present cases for which deterministic models are expected to fail.

4.1. Example 1: Bistable Case. As a first such example we present a coupled system consisting of a bistable type bifurcation ODE with a shallow, double well potential (2.10). Our objective is to allow the stochastic process to induce spontaneous jumps of the hybrid system $(2.1,2.2)$ solution within a reasonable computation time; clearly deterministic closures such as mean field or stochastic averaging could not possibly capture such a solution correctly. Furthermore we could not apply stochastic averaging in this case since it would not be valid for relaxation times which here are as small as $\tau=.001$. In fact, based on our results in [KMS04], we know that for such relaxation times the stochastic noise is substantial thus inducing jumps of the solution between the potential wells. Although we do not examine the phase transition case in the spin flip system, here the behavior seen in our simulations is nevertheless driven by a phenomenon equivalent to phase transitions through the coupling in (2.2) with the external ODE.

More specifically we examine the coupled system (2.2), (2.1) with the following type of bistable bifurcating ODE,

$$
\frac{d X}{d t}=\frac{1}{\tau_{c}}\left(a(\bar{\sigma}) X+\tilde{\gamma} X^{3}\right),
$$


where $a(\bar{\sigma})=z+b(1-\bar{\sigma})$, with $b=4, z=-1$ and $\tilde{\gamma}=-100$. The external potential we apply in this example is linear and has the form, $h=h(X)=c X+h_{0}$ with $c=$ $-1, h_{0}=.005$. Note that as long as $a(\bar{\sigma})>0,(4.1)$ has three nodes: $0, \pm \sqrt{-a(\bar{\sigma}) / \tilde{\gamma}}$; furthermore (4.1) has two main bifurcation states: bistable at $\pm \sqrt{-a(\bar{\sigma}) / \tilde{\gamma}}$ or single node at 0 .

The mean-field formulation and stochastic averaging closure corresponding to the top equation of (3.7) for this bistable example are respectively presented below,

$$
\text { Mean field: } \begin{cases}\frac{d}{d t} Y & =\frac{1}{\tau_{c}}[b(1-\bar{u})+z] Y+\frac{\tilde{\gamma}}{\tau_{c}} Y^{3} \\ \frac{d \bar{u}}{d t} & =\frac{1}{\tau_{I}}(1-\bar{u})-\frac{1}{\tau_{I}} \bar{u} e^{-\beta J_{0} \bar{u}+\beta h(Y)}\end{cases}
$$

$$
\text { Stochastic averaging: } \quad \frac{d}{d t} \bar{x}=\frac{1}{\tau_{c}}\left[b\left(1-u_{\beta, N}(h(\bar{x}))\right)+z\right] \bar{x}+\frac{\tilde{\gamma}}{\tau_{c}} \bar{x}^{3}
$$

Although both the stochastic averaging and mean-field closures are provided above
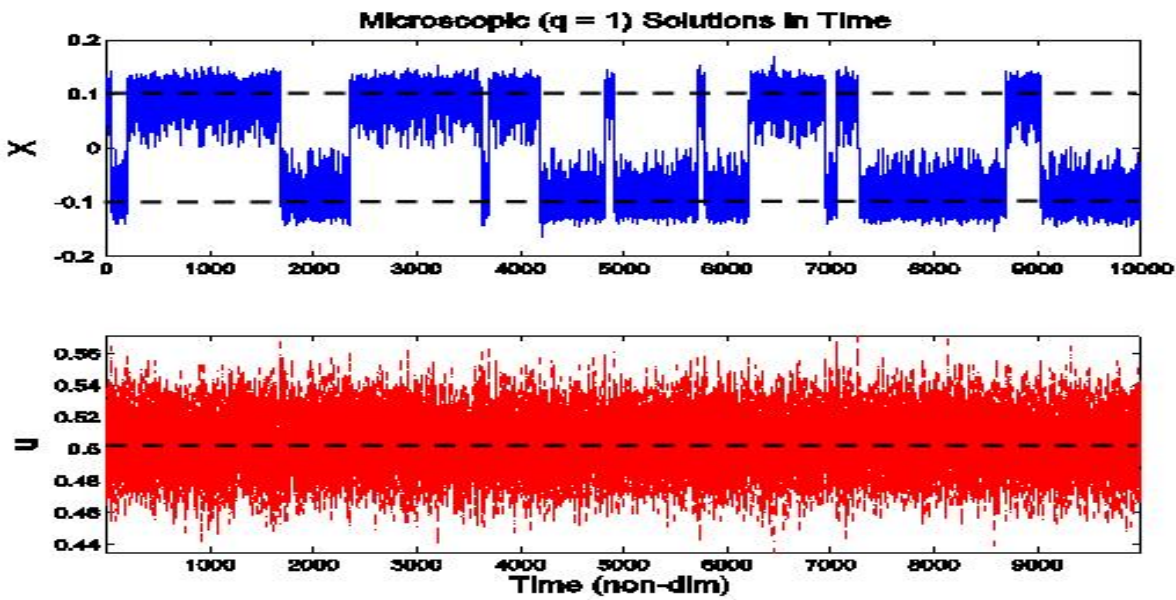

Figure 4. Comparisons for the microscopic $(q=1)$ and meanfield solutions for the coupled system in the (4.1) case. Solutions presented for $\beta J_{0}=.01$ and $\tau=.001$ so that the noise levels are quite high. Other parameters: $L=20, N=1000$ with $b=4, z=$ $-1, \tilde{\gamma}=100, c=-1$ and $h_{0}=.005$.

it is only the mean-field system which is expected to be valid for this example based on the parameter regimes used. The stochastic averaging closure (4.3) would only be valid if $\tau>>1$. The mean field closure (4.2) on the other hand predicts nodes at $Y=0$ and $Y= \pm .1$. We display the solutions for the bistable deterministic/stochastic coupled system versus the mean-field equation in Figure 4 . In general we note that: (a) The deterministic closure attains and remains for all time at the closest, according to dynamics, stable node based on its initial position. (b) Due to the high noise the stochastic process jumps occur almost immediately for the coupled system. The solution switches between the stable nodes of (4.2). Based 
on these findings it is clear that the deterministic closures will fail to capture the transient dynamics which are driven by stochasticity as Figure 4 strongly suggests, although they capture the correct average states. Similar failures are recorded, but not presented here, in the case of the stochastic averaging closure although since $\tau=.001$ this behavior is expected. In the next section we examine how failure of deterministic closures may still occur even though noise levels are not as high.

4.2. Example 2: Saddle Bifurcation. Strong noise is just one of many reasons for which the closures we presented so far may fail. We therefore present below an example where the failure in obtaining the correct system solution is not just attributed to noise but mainly to the occurrence of a rare event which can possibly appear in long enough time when the noise level is low; on the other hand, recall that the deterministic closures derived in Section 3 are valid only for finite times.

The saddle bifurcation example which we examine here has two fixed points which either appear or disappear based on the sign of the stochastic noise parameter $\bar{\sigma}$ which is expressed through the coupling. The specific dynamical system corresponding to $(2.1)$ is,

$$
\frac{d X}{d t}=\frac{1}{\tau_{c}}\left(r(\bar{\sigma})+\tilde{\gamma} X^{2}\right) \text {, where we let } r(\bar{\sigma})=b(z-\bar{\sigma})
$$

We apply the following linear external potential: $h(X)=c X+h_{0}$. As usual all parameters are provided at the tables which follow. Here $b=1, z=.5, \tilde{\gamma}=$ $-.05, c=5, h_{0}=-1, \tau_{I}=1$ and $\beta J_{0}=.01$.

The ODE in (4.4) has a stability profile which depends on the sign of $r(\bar{\sigma})$. In the case of $r(\bar{\sigma})<0$ we obtain one stable and one unstable node at: $\pm \sqrt{-r(\bar{\sigma})) / \tilde{\gamma}}$. If however $r(\bar{\sigma})>0$ then no nodes exist. Last as $r(\bar{\sigma})$ approaches 0 then the two nodes at $\pm \sqrt{-r(\bar{\sigma})) / \tilde{\gamma}}$ eventually merge creating a saddle bifurcation at 0 . In this example we will set $\tau=1$. Although this value is not in the range of $\tau>>1$ the stochastic averaging closure could still be applied successfully based on our experimental simulation findings in [KMS04]. We have similar expectations for the mean field closure. In that respect we present and briefly study both deterministic closures below for the top part of equation (3.7),

$$
\text { Mean field: } \quad\left\{\begin{aligned}
\frac{d}{d t} Y & =\frac{b}{\tau_{c}}[z-\bar{u}]+\frac{\tilde{\gamma}}{\tau_{c}} Y^{2} \\
\frac{d}{d t} \bar{u} & =\frac{1}{\tau_{I}}(1-\bar{u})-\frac{1}{\tau_{I}} \bar{u} e^{-\beta J_{0} \bar{u}+\beta h(Y)}
\end{aligned}\right.
$$

The solutions for both the averaging principle closure and the microscopic coupled system are displayed in Figure 5. A simple numerical study of both the mean field and stochastic averaging above shows that both predict the exact same stable node at $\bar{u} \approx .499, Y \approx .14$. This estimate is in complete agreement with the microscopic simulation of our hybrid system as can be seen in Figure 5. However both of these closures (4.5) and (4.6) are assumed to hold in finite time as also supported by our simulations.

Indeed the values predicted by the stochastic averaging closure are captured in the hybrid simulation as can be seen in the first part of Figure 5 for short times. However for long enough time a rare event jump occurs in the microscopic hybrid system which is not followed by the stochastic averaging or the mean field solution and eventually creates a blow-up as Figure 5 shows. 


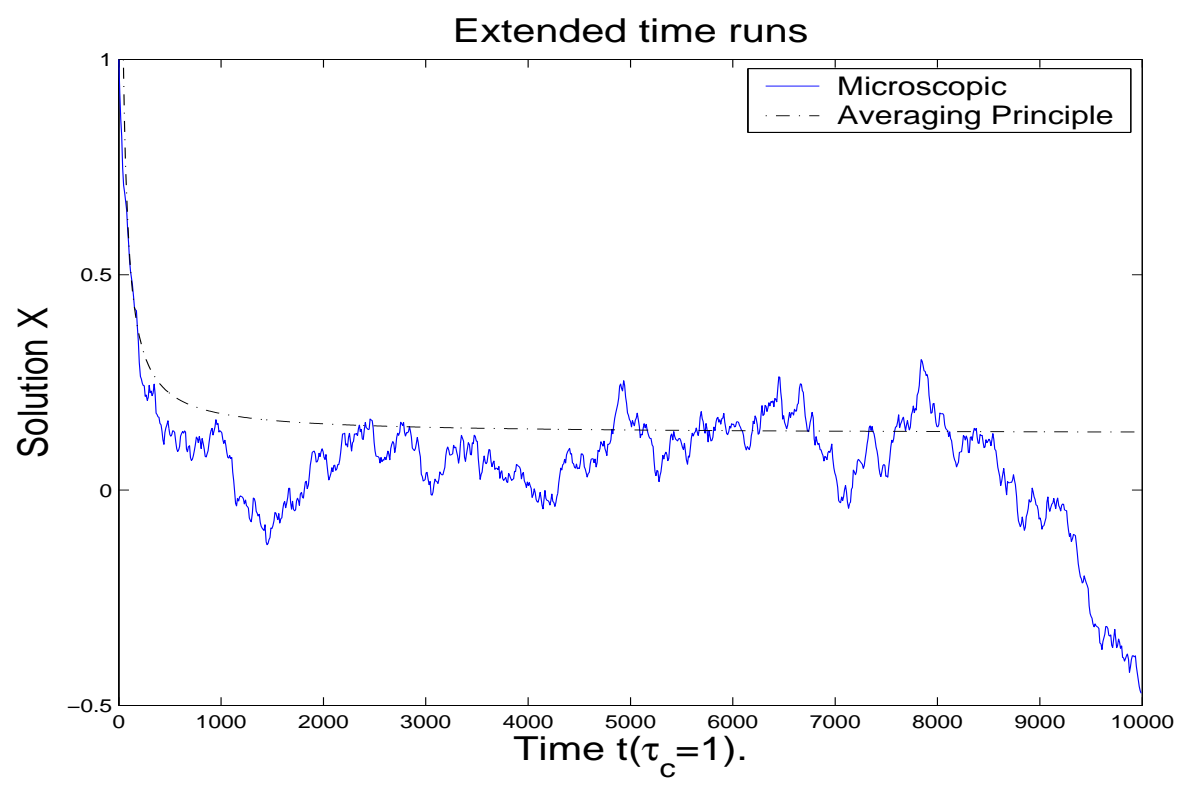

Figure 5. Saddle ODE example. Extended time runs. Solutions for both microscopic Monte Carlo system and stochastic averaging closure. Parameters: $b=1, \tilde{\gamma}=-.05, \tau=1$ and $\beta J_{0}=.01$. Note the rare event jump which eventually drives the system to a finite time blow-up. The stochastic averaging closure is unable to track such a jump.

4.3. Example 3: Hopf Bifurcation. For our last major example in this subsection we examine the hybrid system $(2.1,2.2)$ under a CGL type ODE (2.12):

$$
\frac{d \vec{X}}{d t}=\frac{1}{\tau_{c}}\left[A \vec{X}-|\vec{X}|^{2} \vec{X}\right], \quad \text { where } A=\left(\begin{array}{cc}
a(\bar{\sigma})+\gamma & -\omega \\
\omega & a(\bar{\sigma})-\gamma
\end{array}\right),
$$

$|\vec{X}|^{2}=X^{2}+Y^{2}$ and $a(\bar{\sigma})=b(\bar{\sigma}-z)$ with $b=4$ and $z=.5$. We apply a linear external potential $h(\vec{X})=c\left(X+h_{0}\right)$ with $c=.5, h_{0}=-1$. and choose the remaining parameters as follows: $\omega=1, \tau_{c}=.01, \gamma=.9, \beta=.01, J_{0}=1, X(0)=1, Y(0)=1$. Equation (4.7) has a stable node at $(X, Y)=(0,0)$ for $a(\bar{\sigma})<0$. Otherwise if $a(\bar{\sigma}) \geq 0$ we have a limit cycle with radius depending on the size of $\bar{\sigma}$ (nonsymmetric limit cycles occur for values of $\bar{\sigma}$ approaching 1$)$.

The mean-field equations (3.7) for this hybrid model are

$$
\text { Mean field: }\left\{\begin{aligned}
\frac{d}{d t} Y_{1} & =\frac{1}{\tau_{c}}\left[(a(\bar{u})+\gamma) Y_{1}-\omega Y_{2}-Y_{1}^{3}-Y_{1}^{2} Y_{2}\right] \\
\frac{d}{d t} Y_{2} & =\frac{1}{\tau_{c}}\left[\omega Y_{1}+(a(\bar{u})-\gamma) Y_{2}-Y_{1}^{2} Y_{2}-Y_{2}^{3}\right] \\
\frac{d \bar{u}}{d t} & =\frac{1}{\tau_{I}}\left[1-\bar{u}-\bar{u} e^{-\beta J_{0} \bar{u}+\beta h(\vec{Y})}\right],
\end{aligned}\right.
$$

while the corresponding averaging closure for this case is,

Stochastic Averaging: $\left\{\begin{aligned} \frac{d}{d t} \bar{x}_{t} & =\frac{1}{\tau_{c}}\left[\left(a\left(u_{\beta, N}\left(h\left(\bar{x}_{t}\right)\right)\right)+\gamma\right) \bar{x}_{t}-\omega \bar{y}_{t}-\bar{x}_{t}^{3}-\bar{x}_{t}^{2} \bar{y}_{t}\right] \\ \frac{d}{d t} \bar{y}_{t} & =\frac{1}{\tau_{c}}\left[\omega \bar{x}_{t}+\left(a\left(u_{\beta, N}\left(h\left(\bar{x}_{t}\right)\right)\right)-\gamma\right) \bar{y}_{t}-\bar{x}_{t}^{2} \bar{y}_{t}-\bar{y}_{t}^{3}\right]\end{aligned}\right.$ 

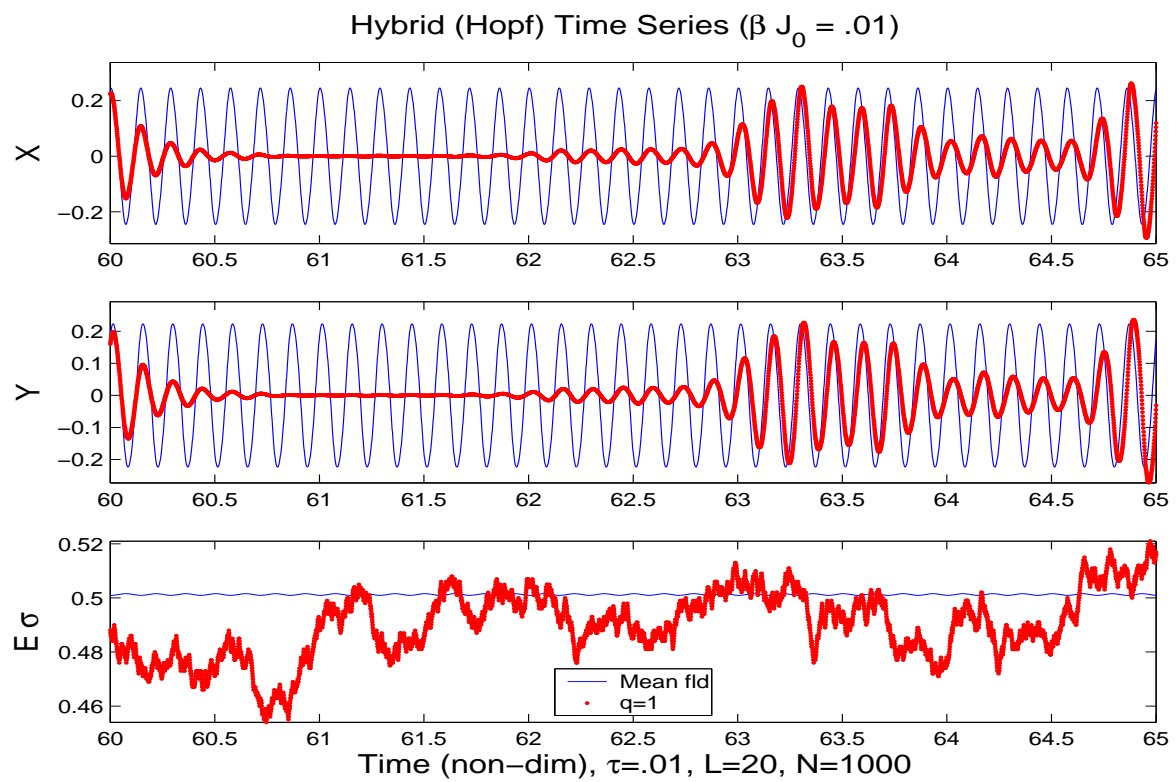

Figure 6. CGL case. The mean field and microscopic hybrid system solutions compared in time. The mean field closure is unable to predict the interchange between the node at $\left(Y_{1}, Y_{2}, \bar{u}\right)$ and random radii limit cycles for this hybrid system. Parameters: $\tau=.01$ and $\beta J_{0}=.01$.

The stochastic averaging closure (4.9) is unable to track the correct behavior for the solutions of the system $(2.1,2.2)$ since the assumption $\tau>>1$ is violated for the parameter regimes of this numerical example. On the other hand the mean field closure (4.8) appears to be more suitable since no timescale separation assumption is required. The mean field closure however reveals constant radius limit cycles centered at $\left(Y_{1}, Y_{2}, \bar{u}\right) \approx(0,0, .5)$; this is also validated from analysis of the Jacobian corresponding to the linearization of (4.8) around such a state, which shows the following eigenvalues $\lambda_{Y_{1}} \approx .05+.41 i, \lambda_{Y_{2}} \approx .05-.41 i$ and $\lambda_{\bar{u}} \approx-2.1$. The mean field closure therefore fails to capture the true behavior of our hybrid system which consists of random interchanges between the node at $(0,0, .5)$ and limit cycles of random radii as can also be seen in Figure 6; this phenomenon is clearly driven by noise which induces transitions through the bifurcation point defined by the sign of $a(\bar{\sigma})$.

4.4. Example 4: FitzHugh Nagumo case. For our last example we chose a phase transition regime $\beta J_{0}=6$ under the linear ODE (2.13). Based on our earlier discussion in Section 3.2 this results in the FitzHugh Nagumo (FHN) coupling (2.13) for our hybrid system $(2.1,2.2)$. Therefore assuming uniform long-range interactions and $L=2 N+1$ while $L, N \rightarrow \infty$ we obtain as in (3.7) the corresponding Curie-Weiss mean field system,

$$
\text { Mean field: }\left\{\begin{aligned}
\frac{d}{d t} Y & =\frac{1}{\tau_{c}}[\omega \bar{u}+b-c Y] \\
\frac{d \bar{u}}{d t} & =\frac{1}{\tau_{I}}\left[1-\bar{u}-\bar{u} e^{-\beta J_{0} \bar{u}+\beta h(Y)}\right]
\end{aligned}\right.
$$



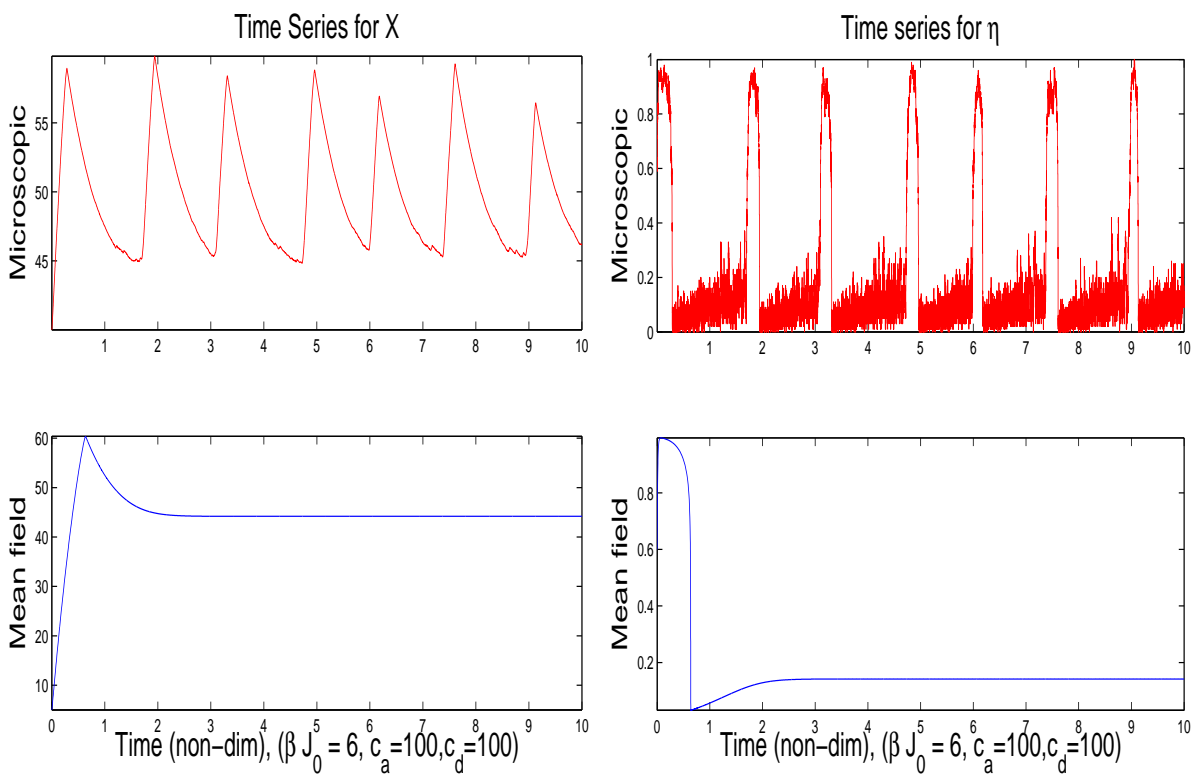

Figure 7. Single excitable stable node exhibiting pulses. Microscopic versus mean field solutions for $X$ and $\eta$ respectively in the phase transition regime: $\beta J_{0}=6$. Other parameters used: $c_{a}=c_{d}=100, a=100, c=1, b=30, \alpha=.01, N=100$ and $\gamma=0$. Initial values $(\bar{\eta}, X)=(.6,40)$.

Details of the derivation can also be found in Section 8.1. The failure of the mean field model (4.10) in this case, will be attributed to critical parameter excitation induced by noise. One important point for the mean field closures is that if the coupling is subjected to high noise levels mean field theories are expected to fail [KMS04]. We choose suitable parameters in (3.7) for which the noise constantly probes the critical values for which bifurcations occur for the coupled system. The completely coupled system therefore can move between regions of stability or instability and even change completely its phase diagram based on the coupling noise parameter $\bar{\sigma}$. The stability behavior which can be expected in the phase transition regime $\beta J_{0}>4$ for (3.8) comprised of:

- Excitable states which can exhibit strong intermittency due to stochastic noise (subplot b, Fig. 2, see also Fig. 7).

- Multiple steady states (subplot c, Fig. 2)

- Oscillatory behavior with random limit cycles as well as random transitions between stable and oscillatory regimes (subplots b, c, d, Fig. 2).

We present both the microscopic and mean field solutions in Figure 7 for both $X$ and $\sigma$ for an example with excitable stability behavior. Note that once again the mean field solution fails to capture the behavior of the microscopic system. Although this system is described by a single stable node the actual solution is perturbed from it in an intermittent manner. More specifically, as can be seen in Figure 7, the microscopic system solution for relatively small $N=100$ displays multiple pulses while the mean field solution is described by a single excitable state. 
In this example the failure is attributed both to the inherent stochastic noise of the system as well as to the phase transition regime which has changed the underlying dynamics as described in Figure $2 \mathrm{~b}$.

\section{Stochastic Closures via Coarse-Graining}

The interplay between scales and spatio/temporal fluctuations, necessitates the consideration of stochastic closures of the hybrid system that would provide accurate and computationally efficient approximations. Motivated by the examples in the previous section where stochastic noise played a crucial role in transient and long-time dynamics we now pursue the derivation of reduced stochastic approximations of the hybrid models that will allow both enhanced computational efficiency but also improved accuracy and predictive capability over deterministic closures. More precisely we obtain stochastic closures of the hybrid system in terms of a coupled ODE / coarse grained stochastic lattice model. Uncoupled stochastic coarse grained closures were first introduced in [KMV03a] and [KMV03b]. Here we extend these methods to hybrid systems and test their validity in the context of the three examples, presented earlier, where deterministic closures fail.

5.1. Coarse Graining of the Hybrid System. In this section we obtain a stochastic closure, defined on the coarse lattice $\mathcal{L}_{c} \subset \mathcal{L}$, where $\mathcal{L}$ denotes the original microscopic lattice. The closure will be valid for all characteristic times $\tau_{c}, \tau_{I}$. Through the coarse graining which we are about to develop here it is possible to extract a closed stochastic coupled system which does not depend on the expected values but instead on the coarse cells themselves. Using the empirical measure $\mu_{N}$ defined in (3.3) as a starting point, we define the coarse grained random process,

$$
\eta(k)=\mathcal{T}(\sigma)(k):=\sum_{x \in D_{k}} \sigma(x), \quad \text { for } \quad k=1, \ldots, n .
$$

and $\eta=\eta(k): k \in \mathcal{L}_{c}=\{1, \ldots, m\}$, with $\eta(k) \in\{0,1, \ldots, q\}$ the coverage of the coarse cell $D_{k}$ for $1 \leq k \leq m$ and $N=(m q)$. Note that each $D_{k}$ consists of $q$ microcells and the order parameter $\eta_{k}$ counts the number of particles in each coarse cell $D_{k}$. Here the coarse lattice $\mathcal{L}_{c}$ is defined through,

$$
\mathcal{L}_{c}=\frac{1}{m} Z \cap[0,1] \text { where } \quad \mathcal{L}_{c} \subset \mathcal{L} .
$$

We define the coarse configuration space $\mathcal{H}_{m, q}=\{0,1, \ldots, q\}^{\mathcal{L}_{c}}$. The projection operator $\mathcal{T}$ is defined implicitly by (5.1). For any test function $g \in L^{\infty}\left(\mathcal{H}_{m, q} ; R\right)$ we have,

$$
f(\sigma):=g(\mathcal{T}(\sigma))=g(\eta)
$$

where $f$ is a test function in $L^{\infty}\left(\mathcal{H}_{N, 1} ; R\right)$. Therefore (2.2) with generator (2.3) can be written as follows in terms of the coarse lattice $\mathcal{L}_{c}$,

$$
\frac{d}{d t} E f(\sigma)=E \sum_{x \in \mathcal{L}} c(x, \sigma)\left[f\left(\sigma^{x}\right)-f(\sigma)\right]=E \sum_{k \in \mathcal{L}_{c}} \sum_{x \in D_{k}} c(x, \sigma)\left[f\left(\sigma^{x}\right)-f(\sigma)\right]
$$

Note that for $x \in D_{k}$ we have,

$$
\mathcal{T}\left(\sigma^{x}\right)(k)=\sum_{y \in D_{k}} \sigma^{x}(y)= \begin{cases}\eta(k)+1 & \text { when } \sigma(x)=0 \\ \eta(k)-1 & \text { when } \sigma(x)=1\end{cases}
$$


Thus the right hand side of (5.3) can be expressed in terms of $g$ as,

$$
f\left(\sigma^{x}\right)-f(\sigma)=(1-\sigma(x))\left[g\left(\eta+\delta_{k}\right)-g(\eta)\right]+\sigma(x)\left[g\left(\eta-\delta_{k}\right)-g(\eta)\right]
$$

where $\delta_{k} \in \mathcal{H}_{m, q}$ denotes the configuration with a single particle at site $k \in \mathcal{L}_{c}$. Rearranging the summations accordingly in the microscopic generator $L$ in (5.3) for $\sigma$ and corresponding coarse state $\eta=\mathcal{T}(\sigma)$ with rates from (2.6) we obtain,

$$
\frac{d}{d t} E g(\eta)=\frac{d}{d t} E f(\sigma)=E \sum_{k \in \mathcal{L}_{c}} c_{a}(k)\left[g\left(\eta+\delta_{k}\right)-g(\eta)\right]+c_{d}(k)\left[g\left(\eta-\delta_{k}\right)-g(\eta)\right]
$$

where $g \in L^{\infty}\left(\mathcal{H}_{m, q} ; R\right)$ is a test function and $c_{a}(k), c_{d}(k)$ denote the exactly coarse grained adsorption and desorption rates,

$$
c_{a}(k)=\sum_{x \in D_{k}} c(x, \sigma)(1-\sigma(x)), \quad c_{d}(k)=\sum_{x \in D_{k}} c(x, \sigma) \sigma(x) .
$$

Note that these rates depend on the microscopic configuration $\sigma$ and not on the coarse random variable $\mathcal{T}(\sigma)$. In order to derive a Markov process for the coarse variable $\eta=\mathcal{T}(\sigma)$ we need to express these rates in terms of $\eta$. Indeed for the adsorption we have,

$$
c_{a}(k)=\sum_{x \in D_{k}} c(x, \sigma)(1-\sigma(x))=\sum_{x \in D_{k}} d_{0}(1-\sigma(x))=d_{0}[q-\eta(k)]
$$

where $d_{0}=1 / \tau_{I}$ and $U_{0}$ denotes the energy associated with the surface binding at $x$, (which for simplicity we take $U_{0}=0$ ). Based on relation (5.5) we can therefore define the coarse grained adsorption rate

$$
c_{a}(k, \eta)=d_{0}[q-\eta(k)]
$$

and expresses the rate by which $\eta(k)$ is increased by 1 . We wish to obtain a similar such relation for the desorption $c_{d}(k)$. Since $U(x)=\bar{U}(k)+O(q /(2 L+1))$ (see [KMV03a]) we have

$$
\begin{aligned}
c_{d}(k) & =\sum_{x \in D_{k}} c(x, \sigma) \sigma(x)=d_{0} \sum_{x \in D_{k}} \sigma(x) e^{-\beta\left(U_{0}+U(x)\right)} \\
& =d_{0} \sum_{x \in D_{k}} \sigma(x) e^{-\beta\left(U_{0}+U(x)\right)}=e^{-\beta\left(U_{0}+\bar{U}(k)+O\left(\frac{q}{2 L+1}\right)\right)} \sum_{x \in D_{k}} d_{0} \sigma(x) \\
& =d_{0} \eta(k) e^{-\beta\left(U_{0}+\bar{U}(k)\right)} \exp \left[O\left(\frac{q}{2 L+1}\right)\right]
\end{aligned}
$$

where

$$
\bar{U}(k)=\sum_{\substack{l \in \mathcal{L}_{c} \\ l \neq k}} \bar{J}(k, l) \eta(l)+\bar{J}(0,0)(\eta(k)-1)-h(\vec{X}) .
$$

and the coarse grained interaction potential $\bar{J}(k, l)$ is computed as average of pairwise interactions between microscopic spins on coarse cells $D_{k}$ and $D_{l}$,

$$
\bar{J}(l, k)=m^{2} \int_{D_{k}} \int_{D_{l}} J(r-s) d r d s
$$

Disregarding the $\exp \left[O\left(\frac{q}{2 L+1}\right)\right]$ term in (5.7) we propose the following approximate desorption coarse grained rate,

$$
c_{d}(k, \eta)=d_{0} \eta(k) e^{-\beta\left(U_{0}+\bar{U}(k)\right)}
$$


Based on rates (5.9) and (5.6) we can now provide the coarse grained Markov process generator for $\eta$ based on (5.4),

$$
L_{c} g(\eta)=\sum_{k \in \mathcal{L}_{c}} c_{a}(k, \eta)\left[g\left(\eta+\delta_{k}\right)-g(\eta)\right]+c_{d}(k, \eta)\left[g\left(\eta-\delta_{k}\right)-g(\eta)\right]
$$

and the corresponding coarse grained dynamics

$$
\frac{d}{d t} E g(\eta)=E L_{c} g(\eta), \quad \text { where } g \in L^{\infty}\left(\mathcal{H}_{m, q} ; R\right)
$$

Thus the hybrid system $(2.1,2.2)$ is approximated by the stochastic closure,

$$
\left\{\begin{aligned}
\frac{d}{d t} \vec{X} & =\frac{1}{\tau_{c}} G(\vec{X}, \bar{\eta}) \\
\frac{d}{d t} E g(\eta) & =E L_{c} g(\eta)
\end{aligned}\right.
$$

where $\bar{\eta}=\bar{\sigma}$. Note that in fact for this approximation to work we only require that $2 L+1>>q$ and not that $N \rightarrow \infty$ or $\tau<<1$ as is the case for the deterministic closures in Section 3; furthermore as in most asymptotics the condition $q<<2 L+1$ will require a relatively modest $q /(2 L+1)$ ratio. We will present further numerical as well as theoretical results showing the capabilities and possible limitations of this stochastic coarse grained closure in Section 7. First we provide below an application from catalysis where such a coarse graining closure can be applied.

\section{An example from catalysis}

We motivate further the coarse grained closure proposed in the previous section for our prototype system by providing a concrete application from catalysis. Applications such as this have partly inspired the choice of the models and the ensuing analysis and simulations discussed in this paper.

Microscopic descriptions of surface mechanisms on catalytic reactors, [ZGB86] involve adsorption, desorption, reaction and surface diffusion processes and can be modeled by stochastic Ising systems with spin flip and spin exchange dynamics, [Lig99]. On the other hand, the gas-phase modeling is based on continuum PDE and includes equations for transport, thermodynamics and gas-phase reactions, [BSL60]. The microscopic stochastic surface processes are coupled to the continuum PDE via boundary conditions and adsorption/desorption rates to and from the surface. One of the first examples of hybrid systems studied in the literature can be found in [VSA90], where only two types of particle species are considered, the reactor is assumed to be well-mixed and the pressure along the catalyst is uniform; such a reactor set-up is referred to as a continuous stirred tank reactor, in which case the gas-phase description reduces to an ODE for the spatially uniform partial pressure $P$ of the reactants in the gas phase:

$$
\frac{d P}{d t}=\frac{P_{0}-P}{\tau}+P^{*}\left(\bar{c}_{d}-\bar{c}_{a}\right) .
$$

where $\bar{c}_{d}, \bar{c}_{a}$ are the spatially averaged microscopic rates for desorption and adsorption of species from and to the catalytic surface respectively and are given by,

$$
c_{d}(x, \sigma)=c(x, \sigma) \sigma(x) \quad \text { and } \quad c_{a}(x, \sigma)=c(x, \sigma)(1-\sigma(x)) .
$$

Here $P_{0}$ is the pressure at the reactor's inlet, $P^{*}$ expresses surface capacity, $\tau$ is an appropriate relaxation time. Additional microscopic mechanisms such as the ones mentioned earlier can easily be included in the model. Finally the partial pressure 
$P$ enters in the external field $h$, hence the microscopic stochastic dynamics are fully coupled to $(6.1)$.

This physical system was first studied in the mean-field (MF) regime in [KSA84], where (6.1) is coupled to an ODE describing the spatially uniform coverage on the catalytic surface. The resulting system of two coupled ODEs predicts oscillations both in the coverage and the pressure but it disregards fluctuations, detailed interactions and spatial inhomogeneities. Comparisons between MF and MC simulations [VSA90] show that in many parameter regimes there is substantial agreement attributed to the coupling with the well-mixed gas-phase; drastic discrepancies are also observed, as well as a variety of dynamic and equilibrium behaviors, depending on the separation of time scales between individual micro-mechanisms and the time scale of (6.1).

Following our exposition in Section 5.1, we will now outline how we can obtain the analogous coarse grained hybrid process for (6.1). The microscopic adsorption and desorption rates in (6.1) are defined as,

$$
\begin{aligned}
& \bar{c}_{a}=\frac{1}{N} \sum_{x \in \mathcal{L}} c(x, \sigma) \sigma(x)=\frac{d_{0}}{N} \sum_{k \in \mathcal{L}_{c}} \sum_{x \in D_{k}} \sigma(x) e^{-\beta\left(U_{0}+U(x)\right)}, \\
& \bar{c}_{d}=\frac{1}{N} \sum_{x \in \mathcal{L}} c(x, \sigma)(1-\sigma(x))=\frac{d_{0}}{N} \sum_{k \in \mathcal{L}_{c}} \sum_{x \in D_{k}}(1-\sigma(x)),
\end{aligned}
$$

where $d_{0}$ is a constant, $c_{a}(k)$ and $c_{d}(k)$ are the adsorption and desorption rates in (5.5) respectively and $U(x)$ is the microscopic potential from (2.7) that includes the external potential coupling term. Following the derivation in Section 5.1 we can approximate $\bar{c}_{a}-\bar{c}_{d}$ in (6.1) as define the coarse grained adsorption and (approximate) desorption rates for this process as,

$$
\bar{c}_{a}(k) \approx \frac{d_{0}}{N} \sum_{k \in \mathcal{L}_{c}}(q-\eta(k)) \quad \text { and } \quad \bar{c}_{d}(k) \approx \frac{d_{0}}{N} \sum_{k \in \mathcal{L}_{c}} \eta(k) e^{-\beta\left(U_{0}+\bar{U}(k)\right)}
$$

respectively with error $O(q / L)$ where $\bar{U}(k)$ corresponds to the coarse grained potential (5.8). The corresponding coarse grained gas phase description for $P$ in (6.1) becomes,

$$
\frac{d P}{d t}=\frac{P_{0}-P}{\tau}+P^{*} \frac{d_{0}}{N} \sum_{k \in \mathcal{L}_{c}}\left(q-\eta(k)-\eta(k) e^{-\beta\left(U_{0}+\bar{U}(k)\right)}\right) .
$$

coupled with the coarse grained dynamics for $\eta$.

\section{Validity Regimes of Stochastic Closures}

In this section we examine the coarse grained closure presented above for both the uncoupled and coupled spin flip dynamics cases and present both theoretical estimates of its capabilities as well as numerical simulations and comparisons against the microscopic hybrid system. There are several interesting issues regarding the extend to which we may apply this coarse graining method in order to approximate a microscopic dynamics process. In the derivation presented in Section 5 we did not make use of any underlying assumptions such as $N \rightarrow \infty$ as in the derivation of the mean field models or an assumption $\tau<<1$ on time scale separation. On the other hand the approximation itself included an error (5.7) which depends on the fraction of coarse graining $q$ over the interaction radius $L$. The main questions which we undertake to answer in this section are: (a) general comparison and capabilities of 
the coarse graining closure for the hybrid system $(2.1,2.2)$ under different parameter regimes (b) how far can we extend the assumption $q<<L$ and maintain a reasonably accurate approximation. We examine these and other similar questions below in the setting of our prototype hybrid system $(2.1,2.2)$ coupled to the ODE cases presented in Section 4 for which the deterministic closures failed.

7.1. Uncoupled coarse grained stochastic dynamics. We begin with a theoretical estimate for the uncoupled dynamics case that gives a first concrete indication regarding the regimes of validity of the method.

First we define the concept of relative entropy that will provide a measure of accuracy for the proposed coarse grained procedure. We consider two probability measures $\pi_{1}(\sigma)$ and $\pi_{2}(\sigma)$ on the discrete state space $\mathcal{H}_{N, 1}$. We further define the relative entropy for these measures as

$$
\mathcal{R}\left(\pi_{1} \mid \pi_{2}\right)=\sum_{\sigma \in \mathcal{S}} \pi_{1}(\sigma) \log \frac{\pi_{1}(\sigma)}{\pi_{2}(\sigma)} .
$$

Using Jensen's inequality we can show that the relative entropy can be thought of as a distance between two measures $\pi_{1}$ and $\pi_{2}$ since the following is true,

$$
\begin{array}{ll}
\mathcal{R}\left(\pi_{1} \mid \pi_{2}\right) & \geq 0 \text { and } \\
\mathcal{R}\left(\pi_{1} \mid \pi_{2}\right) & =0 \text { if and only if } \pi_{1}(\sigma)=\pi_{2}(\sigma) \text { for all } \sigma \in \mathcal{H}_{N, 1}
\end{array}
$$

Note that the relative entropy is not a true metric since $\mathcal{R}\left(\pi_{1} \mid \pi_{2}\right) \neq \mathcal{R}\left(\pi_{2} \mid \pi_{1}\right)$ for all possible measures $\pi_{1}, \pi_{2}$. Nevertheless there is an important inequality which allows us to use the relative entropy as a tool for estimating distance between two measures [CT91],

$$
\mathcal{R}\left(\pi_{1} \mid \pi_{2}\right) \geq \frac{1}{2}\left(\sum_{\sigma \in \mathcal{S}}\left|\pi_{1}(\sigma)-\pi_{2}(\sigma)\right|\right)^{2} \equiv \frac{1}{2}\left\|\pi_{1}-\pi_{2}\right\|_{1}^{2}
$$

In order to compare the process $\{\mathcal{T} \sigma\}_{t \geq 0}$ and $\{\eta\}_{t \geq 0}$ we need to carry out an a priori analysis on the space of all right-continuous paths $\eta_{t}:[0, \infty] \rightarrow \mathcal{H}_{m, q}$ which we denote by $\mathcal{D}\left(\mathcal{H}_{m, q}\right)$. We also denote by $Q_{\sigma_{0},[0, T]}$ the measure on $\mathcal{D}\left(\mathcal{H}_{m, q}\right)$ for the process on the interval $[0, T],\left\{\sigma_{t}\right\}_{t \in[0, T]}$ with the initial distribution $\sigma_{0}$. Similarly $Q_{\eta_{0},[0, T]}^{c}$ denotes the measure on the coarse path space $\mathcal{D}\left(\mathcal{H}_{m, q}\right)$. With a slight abuse of notation we also use $\mathcal{I}_{*} Q$ to denote the projection of the measure $Q$ on the coarse path space, i.e., the exact coarsening of the measure $Q$. Based on these definitions we have,

TheOREM 7.1. Suppose the process $\left\{\eta_{t}\right\}_{t \in[0, T]}$ defined by the coarse generator $L_{c}$ is the coarse approximation of the microscopic process $\left\{\sigma_{t}\right\}_{t \in[0, T]}$ then for any $q<L$ and $N$ where $m q=N$ the information loss as $q / L \rightarrow 0$ is

$$
\frac{1}{N} \mathcal{R}\left(\mathcal{T}_{*} Q_{\mathcal{T}_{*} \sigma_{0},[0, T]} \mid Q_{\eta_{0},[0, T]}^{c}\right)=T O\left(\frac{q}{2 L+1}\right)
$$

We refer to [KT06] for the proof. The relative entropy estimate above demonstrates the limitations of the coarse-graining method since it gives an order one error for nearest neighbor interactions $(L=1)$. This is not surprising in view of wellknown renormalization calculations for the Ising model, as well as explicit numerical comparisons [KMV03a]. On the other hand, (5.7) identifies a small parameter in the coarse-graining process (see [KMV03a] for the rigorous details), namely the 
ratio $q /(2 L+1)$. Although the estimate in Theorem 7.1 is for finite times $[0, T]$ only, and grows with $T$, it is still useful; in the case of phase transitions in the microscopic lattice the estimate ensures numerical accuracy during nucleation, which is typically an initial stage of the evolution, see Figure 4. We refer to simulations partly motivated by our rigorous results in [KPS05] that make precisely this point. In addition we have that, the coarse-grained Gibbs measure which we denote by, $\mu_{m, q}^{c g}$, lies within a controlled error from the exact coarse grained measure [KV03]. More specifically, we easily obtain the equilibrium version of Theorem 7.1, namely

$$
\frac{1}{N} \mathcal{R}\left(\left(\mathcal{T}_{*} \mu^{N}\right) \mid \mu_{m, q}^{c g}\right)=O\left(\frac{q}{2 L+1}\right)
$$

Overall Theorem 7.1 and the Gibbs measure estimate 7.2 imply that the transient, as well as the long time dynamics are expected to be captured accurately by the coarse grained closure. This is also observed in the simulations below in Figures 8 and 9 .

Furthermore, a higher order quadratic estimate can be shown for the weak error between observables for $\eta$ and $\mathcal{T} \sigma$. In particular, we let $\psi \in L^{\infty}\left(S_{c}\right)$ be a test function in the coarse level such that there exist another test function $\phi$ in $L^{\infty}\left(S_{N}\right)$ with the property $\psi(\mathcal{T} \sigma)=\phi(\sigma)$. Then it can be shown [KPS05] that the microscopic process $\left\{\sigma_{t}\right\}_{t \geq 0}$ and the coarse grained analogue $\left\{\eta_{t}\right\}_{t \geq 0}$ satisfy the following weak error estimate,

$$
\left|E_{S}\left[\psi\left(\mathcal{T} \sigma_{T}\right)\right]-E_{S}\left[\psi\left(\eta_{T}\right)\right]\right| \leq C_{T}\left(\frac{q}{L}\right)^{2}, \quad \text { for } 0<T<\infty
$$

where $E_{S}$ is defined for the path conditioned on the initial configuration $\eta_{0}=$ $\mathcal{T} \sigma_{0}=S$ and $C_{T}$ is a constant independent of $q$ and $L$ but depends on $T$. See also [KPS05] for simulations where the second order estimate is presented.

We display numerical comparisons in Figure 8 of the coarse graining closure against the corresponding microscopic dynamics for the uncoupled equation (2.2) under several different levels of coarse grainings. The simulations presented in Figure 8 correspond to single realizations and display: (a) pathwise agreement toward equilibrium between both the microscopic as well as the coarse grained process, (b) the level and effects of noise and most importantly (c) how well is metastability tracked. For these simulations we apply a piecewise constant potential with $L=100$ while the number of particles is $N=1000$. We used the same seed for the random number generator so that we will be able to compare effects attributed to each solution and not to different paths of the stochastic process itself. For further details on the numerical implementations we refer to [KMSb].

Based on the simulations in Figure 8 the coarse graining closure displays remarkable agreement with respect to the microscopic process. The coarse grained closure presented in this figure tracks well not only the path to equilibrium but also the respective noise in time. However this is not entirely surprising due to Theorem 7.1 and (7.2) as well as the large deviation principle demonstrated in [KMV03a] when $N=2 L+1$. The simulations presented above and those which follow for hybrid systems are partly justified by the estimate in Theorem 7.1.

One quantity of interest that can be calculated from the simulations is the mean time to reach for the first time a phase transition. This quantity is calculated as the expected value of the random exit time $\tau_{1}=\inf \left\{t>0: \bar{\sigma}_{t} \geq C^{+}\right\}$where $\bar{\sigma}_{t}$ denotes the total coverage at time $t$ and $C^{+}=.9$ (see Figure 8). We then obtain $\bar{\tau}_{1}=E \tau_{1}$ over 5000 realizations for each level of coarse-graining and calculate the relative 


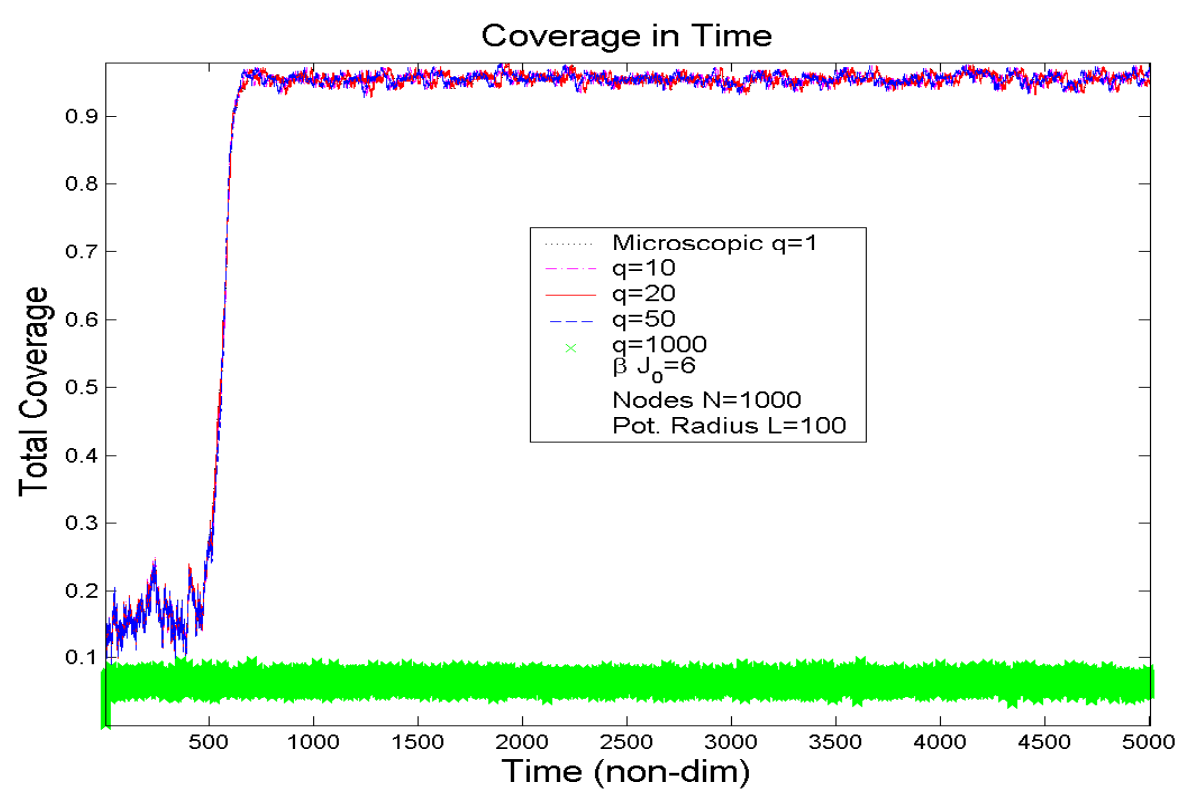

Figure 8. Microscopic versus several different coarse grained dynamics for the uncoupled stochastic model (2.2) in the phase transition regime $\beta J_{0}=6>\beta_{c} J_{0}=4$. The coarse grained dynamics seem to follow the microscopic dynamics to the correct equilibrium dynamics but also capture correctly the noise on its transition path toward equilibrium. Further, all coarse grainings undergo a phase transition at the exact same time instant. As expected the birthdeath case of $q=1000$ does not attain the phase transition (see Section 8.1 for more details on the birth-death process).

\begin{tabular}{|c||rrrrrrr|}
\hline q & 1 & 5 & 10 & 20 & 25 & 50 & 100 \\
\hline \hline Rel. Error \% & 0 & .38 & .82 & 3.42 & 4.91 & 17.69 & 77.73 \\
\hline CPU [sec.] & 309647 & 58412 & 38344 & 16215 & 7574 & 4577 & 345 \\
\hline
\end{tabular}

TABLE 7.2. CPU time and relative error for uncoupled dynamics to reach phase transition. The error is the relative difference between the coarse grained and the exact mean time $\bar{\tau}_{1}$. Simulation and parameters correspond to Figure 8 .

error with respect to the microscopic dynamics. The results of this calculation as well as the particular CPU times corresponding to Figure 8 for each dynamics used are shown in Table 7.1. We further note that the results in Table 7.1 also attest to the fact of the accuracy of the coarse grained dynamics in relation to CPU time speed-up. Table 7.1 shows that we incur less then $5 \%$ relative error for the coarse grained approximation of the phase transition time $\tau_{1}$ corresponding to $q=25$ while, at the same time, we gained a speed-up of approximately 40 times.

The last set of simulations deals with the pathwise behavior on the spatial configuration space for nucleation of a new phase. This is an equivalent simulation 
to that presented in Figure 8 but with $N$ increased to 10000 nodes for higher resolution. We present only qualitative comparison in the series of snap-shots in Figure 9 of the phase transition from the uniform (zero) initial coverage to the full coverage. We observe a striking pathwise agreement on the configuration space for relatively large values of $q$ compared to the interaction radius $L$. However, as the ratio $q / L$ increases, as seen also in Table 7.1, the corresponding coarse-grained process lags behind which is also demonstrated in the expected values of transition times. Such behavior suggests that coarser observables such as switching times in Figure 3 are well-approximated by a highly coarse-grained process while finer resolution is necessary for resolving nucleation of new phases through islands.
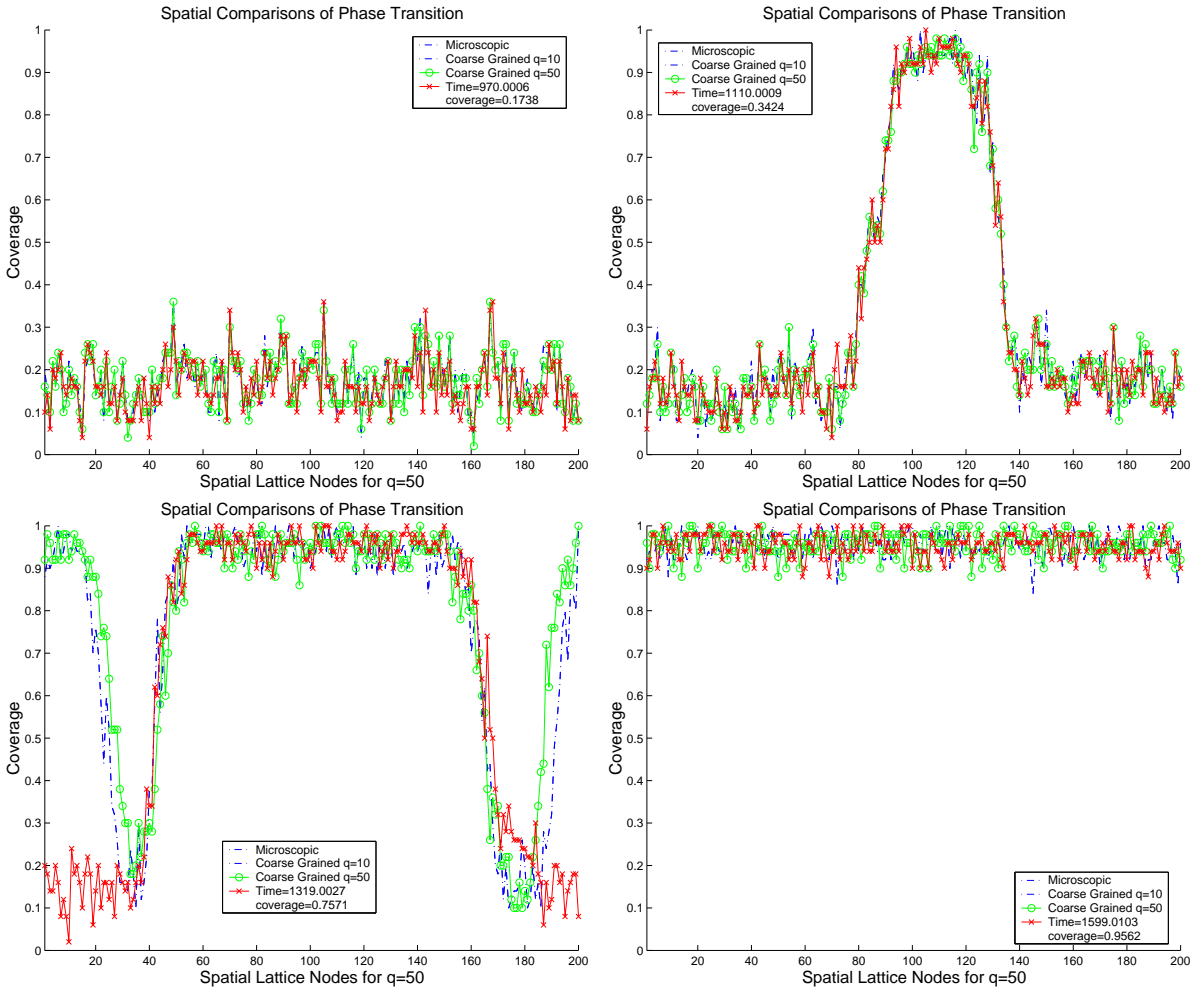

FiguRe 9. Snap-shots of the phase transition starting from zero initial spatial distribution. Comparisons between the microscopic $q=1$ and two coarse grained simulations $q=10$ and $q=50$. The interaction radius is set to $L=200$ while total nodes are $N=10000$. The snap-shots in this simulation correspond to the nucleation phase of Figure 8 with the exception that here we increased the lattice size and potential.

Concluding we remark that the performance during metastability for the uncoupled spin flip system demonstrates the effectiveness of the method both in accuracy as well as in CPU gains in a very demanding set-up. We refer to [KPS05] for further detailed computational comparisons in metastable regimes for (uncoupled) 
stochastic lattice systems, as well as related rigorous time-dependent numerical estimates in the weak topology.

7.2. Stochastic Fluctuations in Coupled Hybrid Systems. In the following subsections we present numerical examples of the coarse graining closure for a number of different types of coupled systems. We examine couplings with ODEs of the type considered in (2.10-2.12). Although a large number of numerical test cases were undertaken only a limited representative number is shown here through tables and figures while our general findings are summarized. We give particular emphasis on the parameter regimes for which the deterministic closures were not able to produce accurate approximations and specifically revisit the related examples from Section 4. Note that we focus on numerical examples with interaction potentials of different sizes $L$ but not $L=1$ (nearest neighbor) since our theoretical results imply more accuracy as $L \rightarrow N$ and $q \rightarrow 1$.

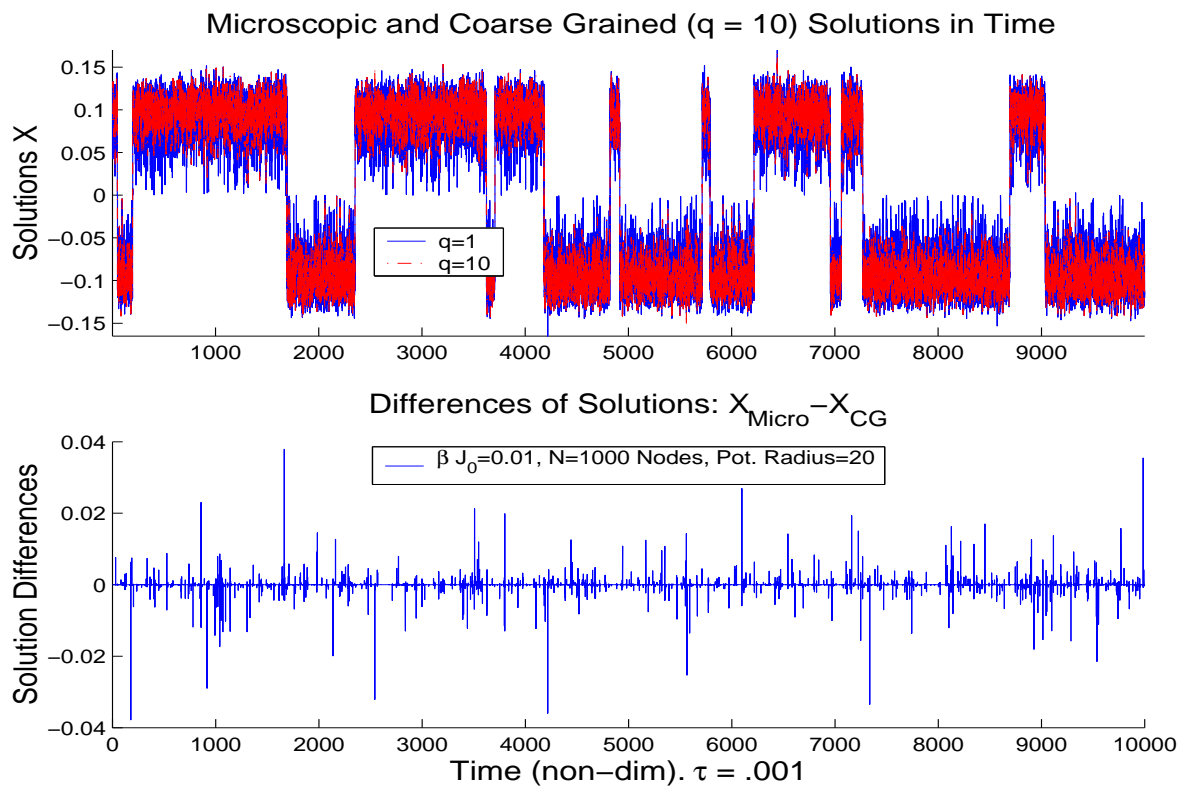

Figure 10. Pathwise comparisons of system $(2.1,2.2)$ for the bistable bifurcation ODE (4.1). We observe complete agreement for the cases presented here of the microscopic and coarse grained $(q=10)$ simulations. Solutions presented for the case of $\beta J_{0}=.01$ and $\tau=.001$ so that the noise levels are quite high. Other parameters: $b=4, z=-1, \tilde{\gamma}=100, c=1$ and $h_{0}=.005$.

7.2.1. Example 1: Bistable Case. Here we re-examine Example 1 from Section 4.1 using the exact same parameters. In that section we carried out comparisons of the microscopic and coarse grained hybrid system solutions for $X$ and $\bar{\sigma}$ corresponding to $\tau=5,1$ and .1 respectively for the case of $\beta J_{0}=.01$ where typically the noise levels are highest. Even though the behavior of the solutions in this example resembles that of a system undergoing phase transitions we are actually away from that regime since $\beta J_{0}<4$. As a result, in this example, we observe/study the significance and influence of noise on the system. 
In the following table we provide further simulation results in terms of $\mathrm{CPU}$ comparisons for the hybrid system above:

\begin{tabular}{|l||c|}
\hline System Type & CPU Time \\
\hline \hline Microscopic & 6695 sec. \\
Coarse Grained at $q=10$ & 237 sec. \\
\hline Coarse Grained at $q=50$ & 44 sec. \\
Coarse Grained at $q=100$ & 28 sec. \\
\hline
\end{tabular}

Note the CPU reduction even for small $q$ values. The horizontal line in the table separating the cases $q=20$ and $q=50$ denotes the point after which we seem to have coarse grained excessively. This is once again in agreement with our theoretical estimate in Theorem 7.1. In fact the cases of $q=50$ and 100 examined numerically in the table above showed progressive deterioration in agreement between the coarse grained and microscopic dynamics. The simulations corresponding to the case of $q=10$ is presented in Figure 10. This case shows excellent agreement between the two dynamics. Similarly we refer to [KMSb] for examples of over-coarse graining displaying the expected deterioration.

7.2.2. Example 2: Saddle Bifurcation. We now re-examine Example 2 from Section 4.2. We will show that the solutions display pathwise agreement between the microscopic and coarse grained Monte Carlo simulation for long time. Note that for this example the stability profile changes every time the stochastic process parameter $\bar{\sigma}$ passes through .5. We run this simulation far enough in time until the rare event which we witnessed before in Section 4 occurs. As expected the rare event jump is leading to finite time blow-up. Remarkably the random jumps for both the microscopic and coarse grained closures occur at almost the same locations.

We compare the solutions of this coupled system in Figure 11 for three different types of coarse grainings: Microscopic $(q=1)$, coarse grained with $q=10,20$. Note that all three simulations fall almost on top of each other with small discrepancies at the very moment that we observe the finite-time blow up.

We summarize the comparisons for the microscopic Monte Carlo simulations for this example against the equivalent coarse grained and averaging principle solutions of (5.12) in the table below. The averaging principle statistics are also included from [KMS04] for comparison purposes. Note the remarkable agreement regardless of relaxation time $\tau$ or value of $\beta J_{0}$ ! All numerics run up to a time of $t=1000$ (or 10000 data samples).

\begin{tabular}{|c|c|c|c|}
\hline $\begin{array}{r}\text { Relative Err } \\
\text { Mici }\end{array}$ & $\begin{array}{l}\text { r Comparis } \\
\text { oscopic vs }\end{array}$ & $\begin{array}{c}\text { ons: } \mid \bar{X}_{M i} \\
\text { veraging }\end{array}$ & $\begin{array}{l}-\bar{X}_{A P}|/| \\
\text { rinciple }\end{array}$ \\
\hline Case & $\beta J_{0}=.01$ & $\beta J_{0}=2$ & $\beta J_{0}=-2$ \\
\hline$\tau=.1$ & $\infty$ & .1 & .71 \\
\hline$\tau=1$ & .87 & .04 & 0 . \\
\hline$\tau=5$ & .08 & .04 & 0. \\
\hline $\begin{array}{r}\text { Relative Err } \\
\mathrm{M}\end{array}$ & $\begin{array}{l}\text { Comparis } \\
\text { croscopic v }\end{array}$ & $\begin{array}{l}\text { ons: } \mid \bar{X}_{M i} \\
\text { Coarse G }\end{array}$ & $\begin{array}{l}-\bar{X}_{C G}|/| \nu \\
\text { rained }\end{array}$ \\
\hline Case & $\beta J_{0}=.01$ & $\beta J_{0}=2$ & $\beta J_{0}=-2$ \\
\hline$\tau=.1$ & - & 0. & 0 \\
\hline$\tau=1$ & .05 & 0. & 0. \\
\hline$\tau=5$ & 0. & 0. & 0. \\
\hline
\end{tabular}




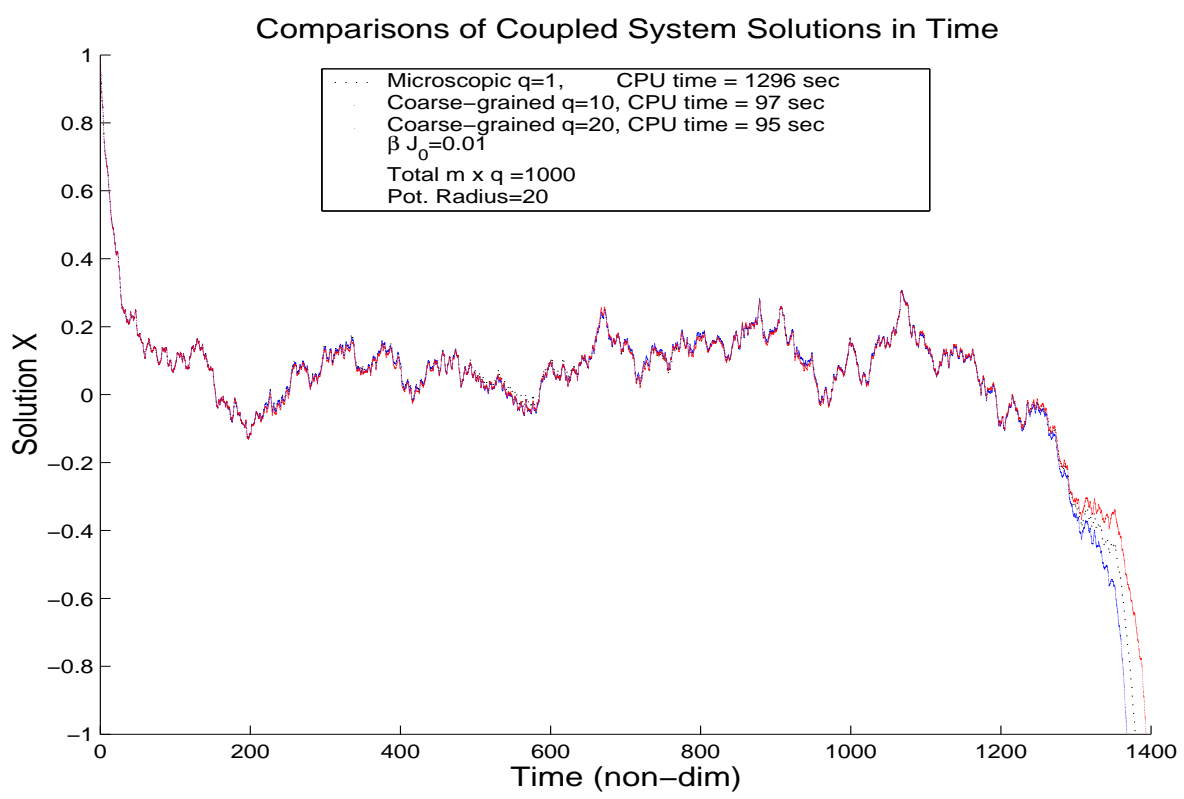

Figure 11. Example of saddle ODE achieving a rare event potential well escape solution for the coupled system. Long time statistics for the case of $\beta J_{0}=.01$ and $\tau=1$.

In general we observe significant agreement between the microscopic and coarsegrained Monte Carlo solutions regardless of relaxation time used as well as significant speed up in CPU time (see Figure 11).

7.2.3. Example 3: Hopf bifurcation. For our last major example in this subsection we revisit Example 3 from Section 4.3 using the exact same parameters as in Section 4.3.

In Figure 12 we display the solutions from the mean field closure, the hybrid system solution $(q=1)$ and the coarse grained solutions for $q=20$ and $q=50$. We observe that once again the coarse grained closure captures correctly the behavior of our hybrid system. Furthermore we show numerical evidence of over-coarse graining in the case of $q=50$ where we observe a small deterioration in the approximation of the solution in accordance with the spirit of Theorem 7.1.

Last we also present CPU comparisons for different coarse graining values for this example in the following table:

\begin{tabular}{|l||ccc|cc|}
\hline Coarse Graining & Microscopic & $\mathrm{q}=10$ & $\mathrm{q}=20$ & $\mathrm{q}=50$ & $\mathrm{q}=100$ \\
\hline \hline CPU Time & $10225 \mathrm{sec}$ & $451 \mathrm{sec}$ & $201 \mathrm{sec}$ & $106 \mathrm{sec}$ & $95 \mathrm{sec}$ \\
\hline
\end{tabular}

The vertical line between $q=20$ and $q=50$ denotes that we have coarse grained too much, according to the results in Theorem 7.1. Surprisingly however, even though we over-coarse grained relative to $L=20$, the case $q=50$ seems to also approximate well the microscopic dynamics as can be seen in Figure 12.

REMARK 7.2. We can also carry out detailed statistical analysis including power spectra, auto- and cross- correlations for the microscopic and the coarse grained time series. However here the results are conclusive since we have demonstrated 

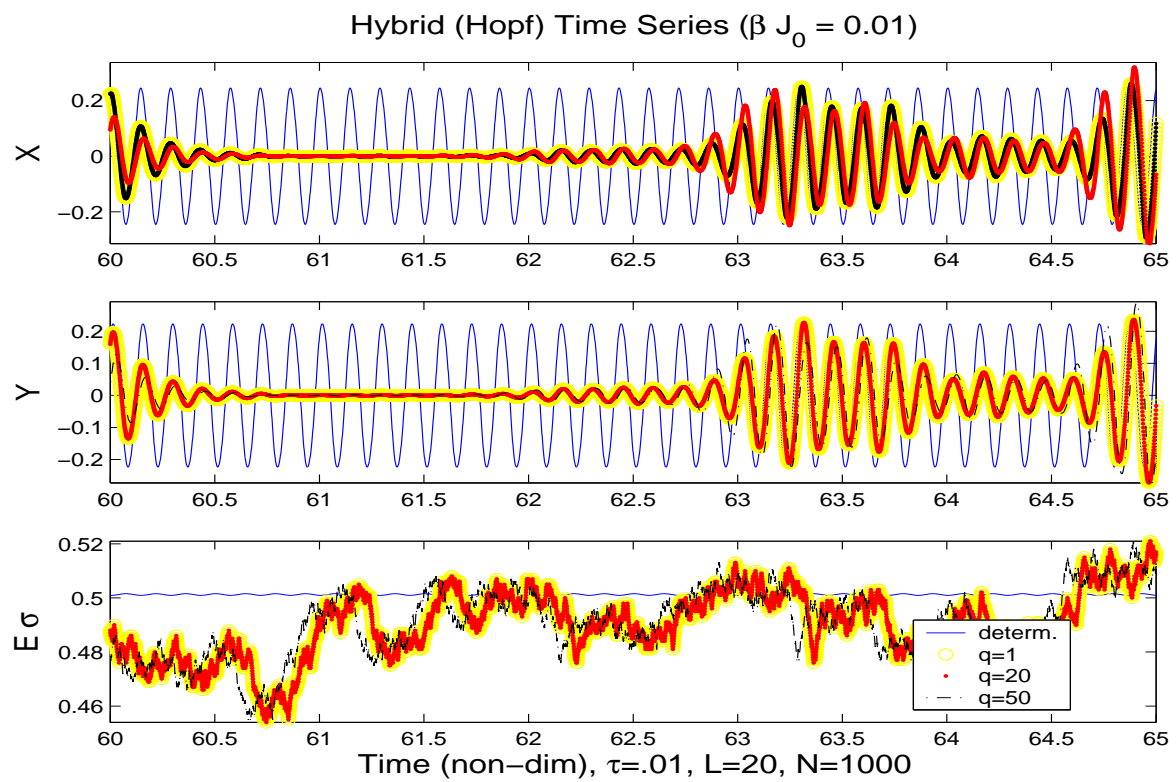

Figure 12. Saddle ODE: Comparisons between the solutions of our hybrid system (5.12) and the mean field closure, microscopic (4.7), coarse grained closure for $q=20$ and (over) coarse grained closure for $q=50$. Same parameters as in Figure 6: $\tau=.001$ and $\beta J_{0}=.01$.

pathwise agreement between microscopic and coarse grained dynamics. On the other hand we refer to the next section (see also [KMSa]) where such a statistical analysis is undertaken for more challenging examples exhibiting intermittency and random oscillations in the presence of phase transitions in the microscopic dynamics. Some calculations and simulations in this direction are presented next.

\section{Intermittency, metastability and phase transitions}

In this section we study the intrinsically complex case where randomness, microscopic interactions and macroscopic dynamics compete and dramatically affect the hybrid system behavior. Specifically, we examine the influence of phase transitions and metastability in the noisy micro-/sub- grid scale on the overall dynamics in the context of our simple prototype hybrid system. Examples of hybrid systems such as $(2.1,2.2)$ and scalar equations with bistable behavior or saddle node bifurcations, as well as a spatially homogeneous complex Ginsburg-Landau equations exhibiting Hopf bifurcations have been presented here but also studied in [KMSb] in regimes without phase transitions in the lattice system. In contrast to these studies, if $\bar{\sigma}$ is constant in (2.1), the resulting ODE is linear with trivial behavior, without any external bifurcations. We therefore focus here on the case of strong interaction / low temperature case where phase transitions occur in the stochastic system.

The FitzHugh Nagumo example (2.13) is chosen as a rich in behavior numerical test case while studying the general behavior under phase transitions for our hybrid 
system $(2.1,2.2)$,

$$
\begin{aligned}
\frac{d}{d t} X & =\frac{1}{\tau_{c}}(\omega \bar{\sigma}+b-c X) \\
\frac{d}{d t} E f(\sigma) & =E L f(\sigma) .
\end{aligned}
$$

We employ different methods for comparing and understanding the solutions of the numerical examples presented here against the microscopic hybrid system. In some cases we carry out visual comparisons of the solutions. However, in the intermittent example pulses occur instantaneously, are short lived, and are harder to compare against the microscopic solution. We therefore also provide a detailed statistical signal analysis for chosen interesting examples which further validate our findings. All such analysis which consists of auto-correlations, periodograms and cross-correlations is carried out on very long signals although in the figures we only display a representative small part.

In the derivation presented in Section 5 we did not make use of any underlying assumptions such as time scale separation (see [KMS04]), or $N \rightarrow \infty$, as is usually the case in the derivation of the deterministic type closures. On the other hand the coarse grained approximation itself includes an error (5.7) which depends on the fraction of coarse graining $q$ over the interaction radius $L$.

The main questions which we address in this section are as follows: we compare different statistical observables (auto-correlations, periodogram, cross-correlations) for various coarse grainings against the hybrid system $(2.1,2.2)$ under different parameter regimes in order to assess the validity of the coarse graining approach; we further probe numerically the extent to which we can push the assumption $q<<L$ (or even $q=L$ ) and maintain a reasonably accurate approximation.

We examine these and other similar questions below in the setting of our prototype hybrid system $(2.1,2.2)$ under selected examples. Although a number of numerical test cases were undertaken only a limited representative number is shown here through tables and figures while our general findings are summarized.

First, however, we start our presentation in Section 8.1 with an example of a coarse graining closure which is exact. This will be the Curie-Weiss system which implies long, weak interaction potential with finite range $L=2 N+1$.

8.1. An exact stochastic closure: the Curie-Weiss system. The purpose of this section is to study the role and influence of microscopic noise and interactions in the overall system dynamics. This is related to Section 4.4. The uncoupled Curie-Weiss spin model (4.10) with a uniform interaction potential $J(x-y) \equiv$ $J_{0}$ for all $x, y$ on the microscopic lattice, is a classic simple example in statistical mechanics that exhibits phase transitions even in one space dimension and allows for detailed explicit calculations such as mean field and thermodynamic limits at all temperatures, [Ell85, LB99]. Here we introduce an analogue of the Curie-Weiss spin system for the hybrid systems $(2.1,2.2)$. As discussed in Sections 3.2 and 4.3 such a system gives rise to the mean field ODE (4.10) in the $N \rightarrow \infty$ limit. We will first examine in this simplest context the influence of noise and phase transitions on the overall hybrid system dynamics by comparing it against the mean field closure (3.7) presented in Section 3. The examples which we present in this section underline some of the limitations of deterministic closures and at the same 
time motivate the numerical simulations of the stochastic closure in the following Section 7 which decidedly resolve the aforementioned shortcomings.

Based on the assumption of uniform particle/particle interactions and the coarse grained closure presented in Section 5 we derive here an exact birth-death type stochastic closure for the Curie-Weiss hybrid system in the sense that there is no error in the approximation: we revisit the coarse grained closure derived in Section 5.1 and especially the error term in the desorption rate $c_{d}(k)$ which is responsible for this closure being an approximation in the first place. Then we have from (5.7),

$$
\begin{aligned}
c_{d}(k) & =\sum_{x \in D_{k}} c(x, \sigma) \sigma(x)=d_{0} \sum_{x \in D_{k}} \sigma(x) e^{-\beta\left(U_{0}+U(x)\right)} \\
& =d_{0} \sum_{x \in D_{k}} \sigma(x) e^{-\beta\left(U_{0}+U(x)\right)}=d_{0} e^{-\beta\left(U_{0}+\bar{U}(k)\right)} \sum_{x \in D_{k}} \sigma(x) \\
& =d_{0} \eta(k) e^{-\beta\left(U_{0}+\bar{U}(k)\right)}
\end{aligned}
$$

since $J(x-y) \equiv J_{0}$ and $U(x)=\bar{U}(k) \forall x \in D_{k}$ which is a direct consequence of the definitions for $U(x)$ and $\bar{U}(k),(5.8)$ and (2.7), respectively. In this case the coarse grained desorption rate above is an exact coarse graining of the original microscopic system. We therefore have the following exact adsorption and desorption rates respectively, at any coarse level $q$,

$$
c_{a}^{b d}(k, \eta)=d_{0}[q-\eta(k)] \quad \text { and } \quad c_{d}^{b d}(k, \eta)=d_{0} \eta(k) e^{-\beta\left(U_{0}+\bar{U}(k)\right)}
$$

Although the calculation is exact for any $q$, we will take $q=N$ since it is the easiest case to implement numerically. In this case we have a single coarse cell and the only order parameter is the total coverage $\eta$ with rates which simplify even further,

$$
c_{a}^{b d}(\eta)=d_{0}[q-\eta] \text { and } c_{d}^{b d}(\eta)=d_{0} \eta e^{-\beta\left(U_{0}+\bar{J}(0,0)(\eta-1)-h(X)\right)} .
$$

The resulting process is a Markov birth-death process with a maximum population $q=N$. Based on rates (8.4) we can now provide the birth-death Markov process generator for $\eta$,

$$
L_{c}^{b d} g(\eta)=c_{a}^{b d}(\eta)[g(\eta+1)-g(\eta)]+c_{d}^{b d}(\eta)[g(\eta-1)-g(\eta)]
$$

where $g \in L^{\infty}\left(\mathcal{H}_{1, q} ; R\right)$. Therefore the exact birth-death closure corresponding to the microscopic system $(2.1,2.2)$ is,

$$
\text { Birth-death closure: }\left\{\begin{aligned}
\frac{d}{d t} X & =\frac{1}{\tau_{c}} G(X, \eta) \\
\frac{d}{d t} E g(\eta) & =E L_{c}^{b d} g(\eta)
\end{aligned} \quad \text { where } g \in L^{\infty}\left(\mathcal{H}_{1, q} ; R\right)\right.
$$

Note further that in the case of $N \rightarrow \infty$ (8.6) easily yields the mean field closure (3.7), by employing the corresponding martingale problem, see for instance [KMV03b, KMS04].

We compare below system $(2.1,2.2)$ to $(3.7)$ for the FitzHugh Nagumo model, $G(X, \eta)=\omega \eta+b-c X$, in the Curie-Weiss case. The birth-death process $\eta$ is used as a computational tool in this comparison since it is an exact coarse graining of the microscopic hybrid model.

8.1.1. Oscillatory regime. We choose parameters, shown in the figures, for our hybrid system $(2.1,2.2)$ which create a single unstable node in the corresponding mean field equation (3.7). We therefore expect oscillatory solutions in Figure 13 for the birth-death process since the parameters chosen produce an unstable single node for the corresponding mean field equation (3.7); the latter, according to the Poincaré-Bendixson theorem will have oscillatory solutions (also compare with Figure 6.8 in [Mur89]). 
Time Series for $\mathrm{X}$

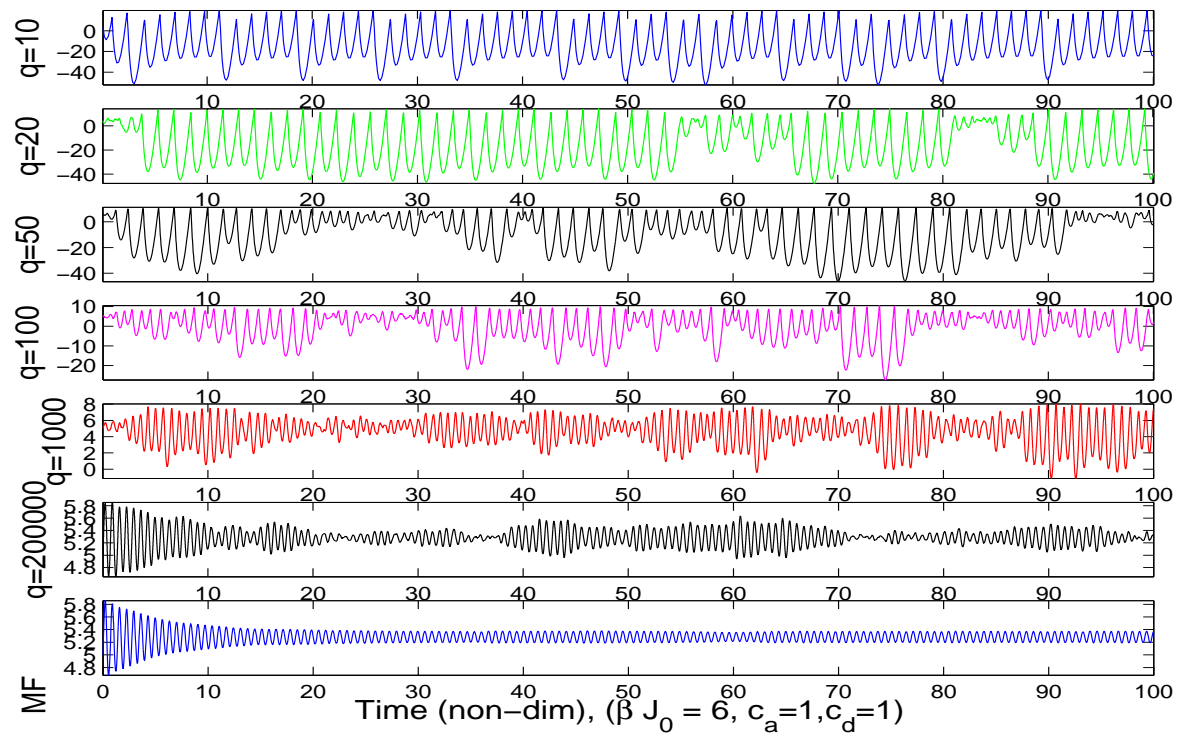

Figure 13. Oscillatory behavior. Comparisons of birth-death and mean field solutions for $X$ under the phase transition regime, $\beta J_{0}=$ 6. Parameters used: $c_{a}=1, c_{d}=1, a=350, c=1, b=-200, \alpha=$ .1 and $\gamma=0$. Initial values $(\bar{\eta}, X)=(.6,5)$.

We display a representative example for the oscillatory case where disagreement is evident when compared against the mean field solution in Figure 13. The two closures differ the most based on the size of the noise or respectively size of $q$.

For all simulations undertaken for this oscillatory example, not all of which are shown here, we found that the Curie-Weiss hybrid system (computed exactly via the birth-death process $\eta$ ) did not always produce solutions close to the mean field deterministic closure solutions (4.10). This is a striking finding and shows that although we expect the birth-death closure to produce the same solutions as the mean field in the limit of $N=q \rightarrow \infty$ in reality, based on large numerical computations, we obtain disagreements between the two even for very high values of $q$, at least in some parameter regimes that appear more sensitive to noise.

8.1.2. Bistable regime. For this example we choose parameters for which there are three nodes in the mean field equation (3.7): two stable and one unstable. This situation gives rise to more than just multiple solitons or pulses for our hybrid system since now the solutions visit the stable nodes and stay there for a random amount of time. See Figures 14 and 15.

We observe that for low values of $q$ (high noise) random departures occur for the birth-death model from either of the two stable nodes. As expected the high $q$ case agrees with the deterministic mean field model. Here the agreement is observed for the low value of $q=1000$ in contrast to results in Section 4.1.

8.1.3. Excitable/intermittent regime. For the parameter regime, provided in Figures 16 and 17, the corresponding mean field system (3.7) displays only one stable node as can be seen in Figure 2b. In due time therefore every solution will 


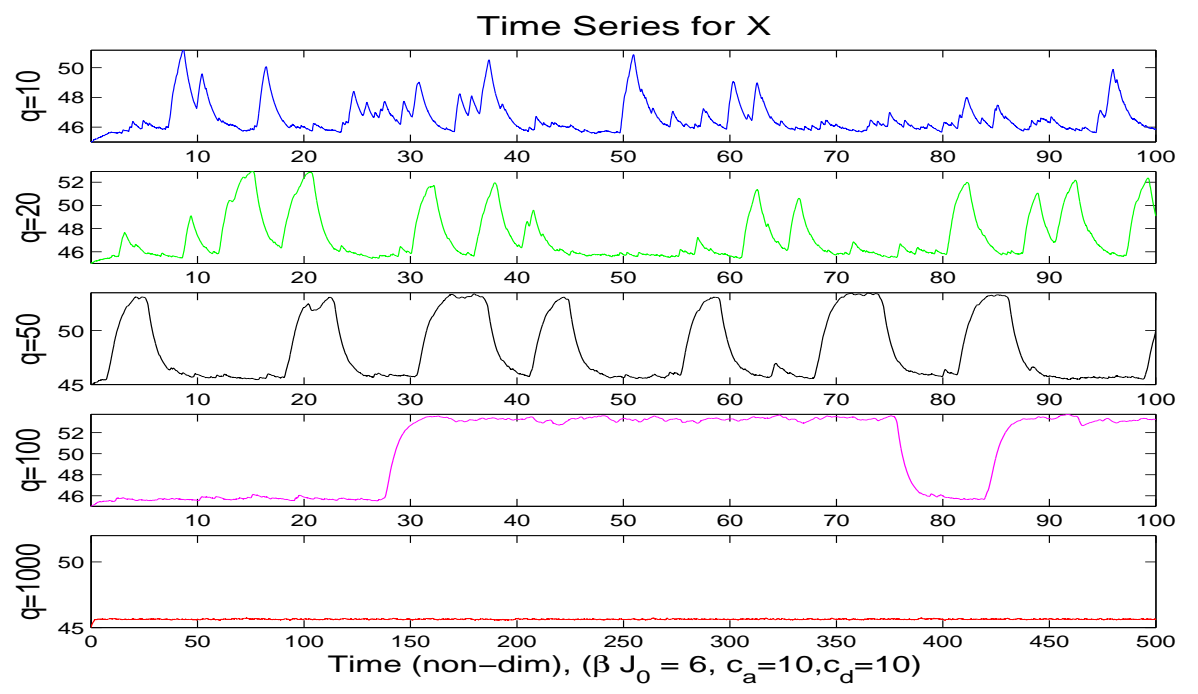

Figure 14. Two stable nodes. Birth-death for $q=10,20,50,100$ and 1000. Coupled system behavior for the phase transition regime: $\beta J_{0}=6$. Other parameters used: $c_{a}=c_{d}=10, a=$ $10, c=1, b=44.5, \alpha=.01$ and $\gamma=0$.

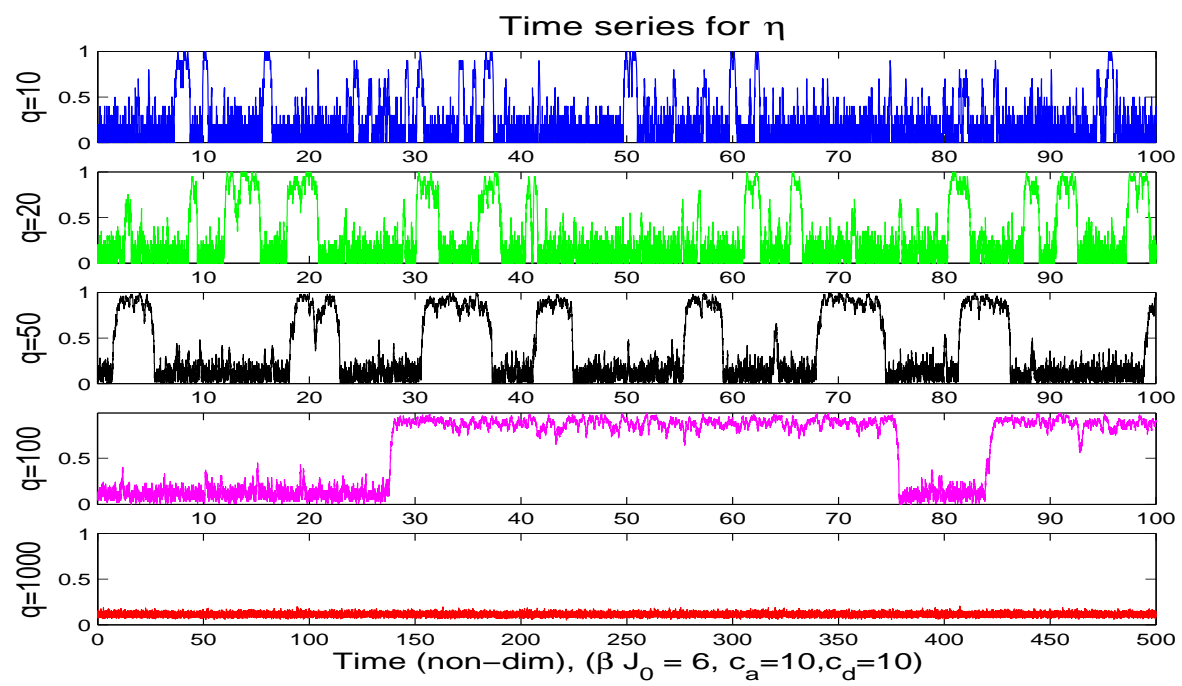

Figure 15. Two stable nodes. Birth-death for $q=10,20,50,100$ and 1000. Coupled system behavior for the phase transition regime: $\beta J_{0}=6$. Other parameters used: $c_{a}=c_{d}=10, a=$ $10, c=1, b=44.5, \alpha=.01$ and $\gamma=01$.

converge to that node although in the process large excursions from this adjustment process can also be recorded. In the hybrid system, this allows for solitons and excitable states to occur due to noise and fluctuations. Furthermore it is possible that 


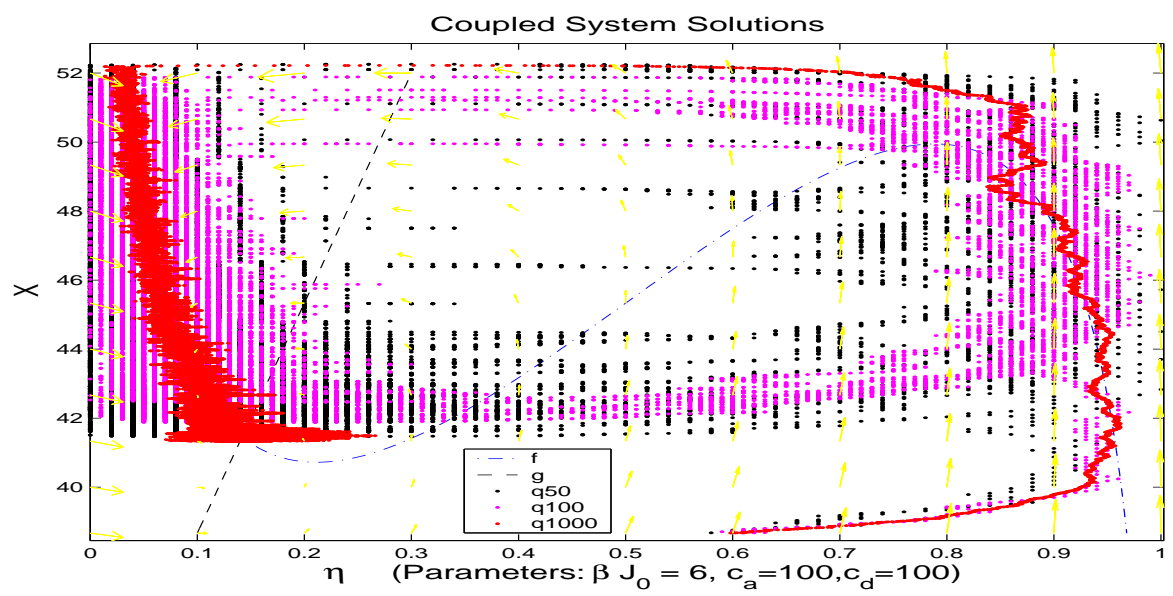

Figure 16. Single excitable stable node with multiple pulses. Birth-death for $q=50,100$ and 1000. Coupled system behavior for the phase transition regime: $\beta J_{0}=6$. Other parameters used: $c_{a}=c_{d}=100, a=100, c=1, b=30, \alpha=.01$ and $\gamma=0$.

some of these solutions keep pulsating randomly due to the underlying stochastic noise in our system thus creating new solitons and an overall strongly intermittent behavior. The overall behavior under this example is equivalent to Example 4 which we examined earlier in Section 4.4. Also compare the results above with Figure 12.6b of pg. 328 in [Mur89].

We display only one example in Figures 16 and 17. In these figures we observe that multiple pulses are possible for low q values (or high noise levels as in Figure 7, Section 4.4). The special example of Figures 16 and 17 exhibits various types of stochastic oscillation which for the case of $q=100$ is almost periodic. The periodicity of the random oscillations suggest the existence of a stochastic resonance regime. In fact we have numerically obtained multi-peaked residence time distributions similar to the ones obtained in systems exhibiting stochastic resonance ([GHJM98], Sec IIB). On the other hand note that typically the birth-death solutions for $q=1000$ display a single pulse and subsequent approach to the stable node as in the FitzHugh Nagumo (FHN) case [Mur89]. In large time intervals however random excitations and rare events will occur [KMSb] which in our simulations here are not observable for the given finite time but also since for higher values of $\mathrm{q}$ the noise levels are lower.

8.2. Non-uniform microscopic interactions. Therefore, based on this discussion and the examples presented, we observe once again that the mean field closure cannot capture the complete transient and long time dynamics. We therefore next examine and present numerical simulations and comparisons between the microscopic couled system and the coarse graining closure for $L<<N$.

8.2.1. Example 1: oscillatory regime. In this subsection we examine our hybrid system $(2.1,2.2)$ under the phase transition regime $\beta J_{0}=6$ for the case that the mean field ODE (3.7) displays oscillatory behavior. We apply similar parameters as those chosen for the equivalent oscillatory example in Section 8.1. In the example 
Time Series for $X$

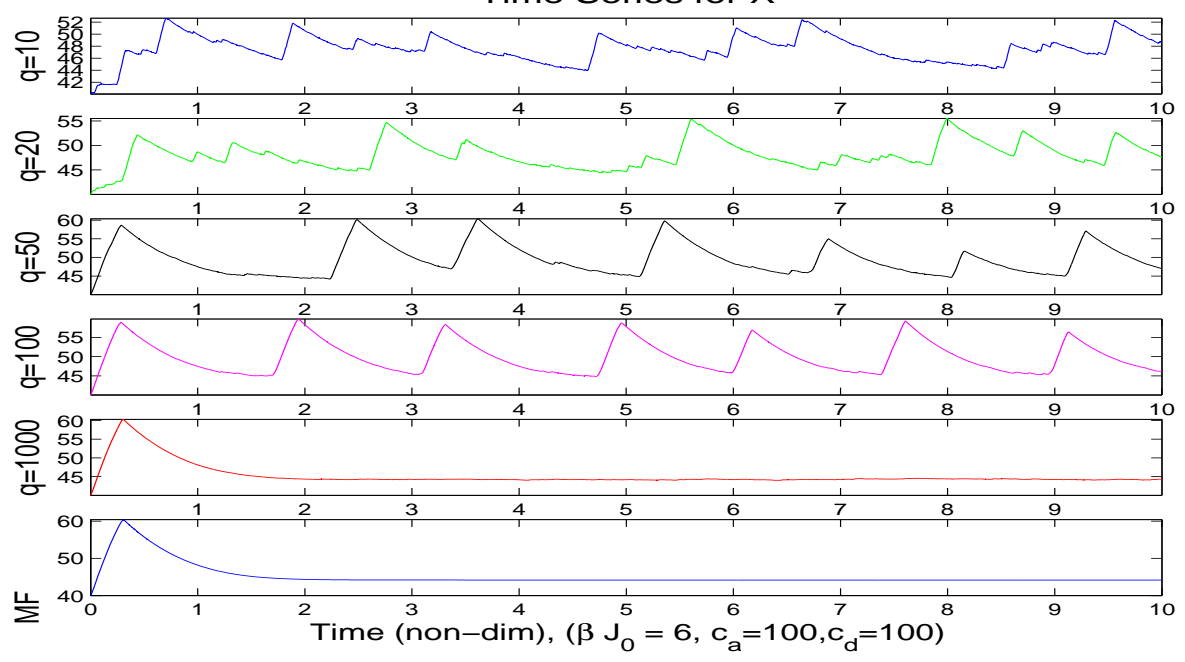

Figure 17. Single excitable stable node with multiple pulses. Birth-death and mean field solutions for $X$ in the phase transition regime: $\beta J_{0}=6$. Other parameters: $c_{a}=c_{d}=100, a=$ $100, c=1, b=30, \alpha=.01$ and $\gamma=0$.

cases which follow in this subsection we examine both long and short range potentials and their effect in the hybrid coupling. We also compare snapshots in time of the spatial distribution of the stochastic model after each example presented.

We first examine the solutions of our hybrid system in the oscillatory regime for a relatively long potential range of $L=200$ while we keep the lattice size at $N=1000$. The time series solution for $X$ is provided in Figure 18 while the corresponding spatial distribution of the spins for selected snap-shots can be found in Figure 19. All parameters are provided in these figures.

Overall we observe a marked difference between the two simulations which is attributed to the size of $L$. As expected, based on our Theorem 7.1, the effect of the size of the potential range is important. Although the agreement is quite good in time but also in space between these solutions our comparisons show that the error in the case of $L=20$ is higher, when compared against the microscopic solution since $q$ is very close to the size of $L$.

Furthermore, under several examples not all presented here, we note that the spatial distribution of the stochastic model takes the form of a sinusoidal wave. This is also visible in Figure 19 here. In the corresponding spatial distribution we note that we do obtain the correct averaged behavior for the coupled system in time although the underlying spatial behavior is quite different! Specifically we obtain the correct average coverage, which is around .5, for this examples as can be seen in Figure 19.

The signal analysis undertaken comparing the microscopic system solution to the corresponding coarse grained solutions for $q=10$ and $q=50$ under a potential range of $L=100$ reveals the following: 
$q=10$ : in this case we verify once again that we have excellent agreement between coarse grained and microscopic dynamics solutions by studying the respective periodogram for each example.

$q=50$ : similarly good agreement is seen for this case when compared with the microscopic dynamics in Figures 20 and 21. The agreement in this case is not supposed to be as good as in the case of $q=10$ since $q=50$ is considered to be high compared to the potential radius of $L=100$.

Spatial: The ensemble periodogram presented in Figure 21 displays remarkable agreement in the frequency space.

The respective cross-correlations between the solutions $X$ and $\eta$ for each dynamics considered have also been studied and seem to be highly correlated under all dynamics, $q=1,10$ and 50, presented.

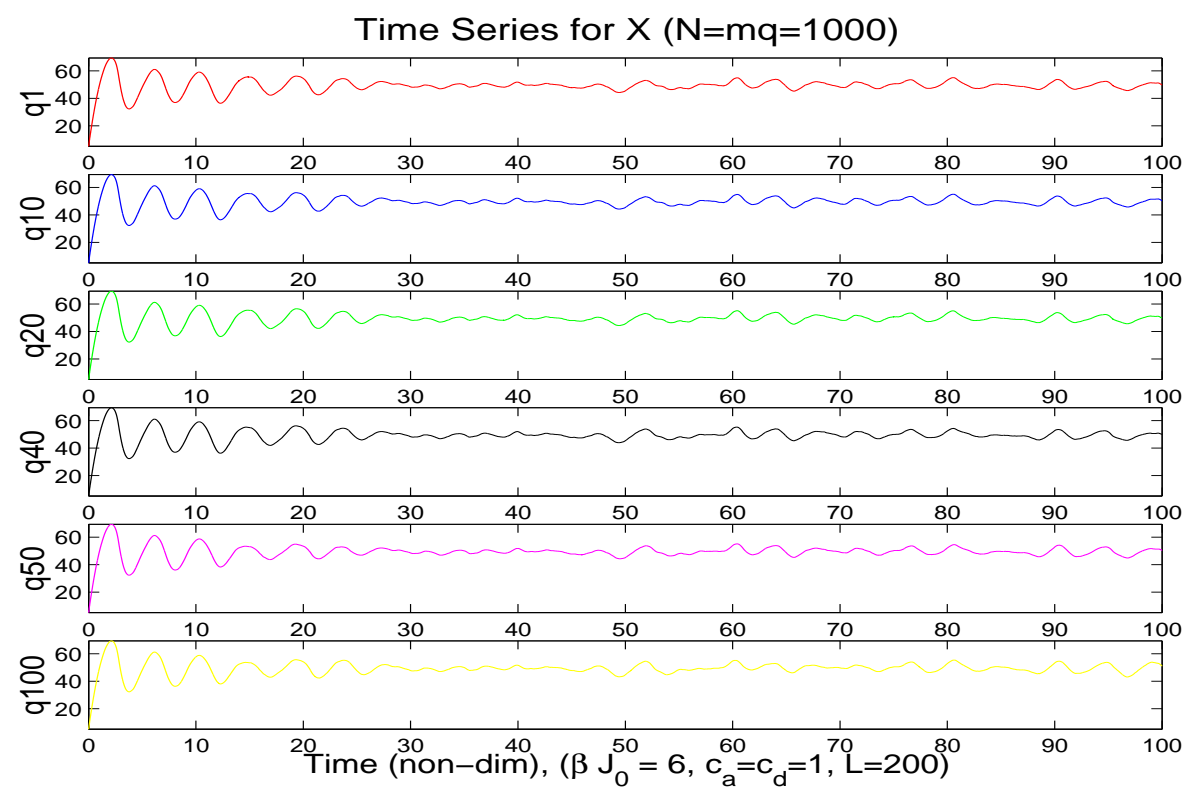

Figure 18. Oscillatory example for $L=200$. Time series for $X$ for microscopic $q=1$ and $q=10,20,40,50,100$ under phase transition: $\beta J_{0}=6$. Other parameters: $c_{a}=c_{d}=1, a=100, b=$ $0, c=1, \alpha=.01, \gamma=0$ and $N=1000$.

8.2.2. Example 2: excitable regime. We now examine the excitable ODE case for our hybrid system $(2.1,2.2)$ under the phase transition regime $\beta J_{0}=6$ with the same parameters as those chosen for the examples in Section 8.1. The parameters are chosen in such a way so that the hybrid system remains in the excitable/intermittent regime producing a pulsating phenomenon. As usual we compare the time series as well as spatial snapshots in time of the distribution of the stochastic model.

We display snapshots of the spatial distribution in Figure 22 while the time series behavior is shown in Figures 23 and 24. The parameters as usual are provided in the figures. Note that in this example we have the phenomenon of random departures. We choose this example since the disagreement between microscopic 

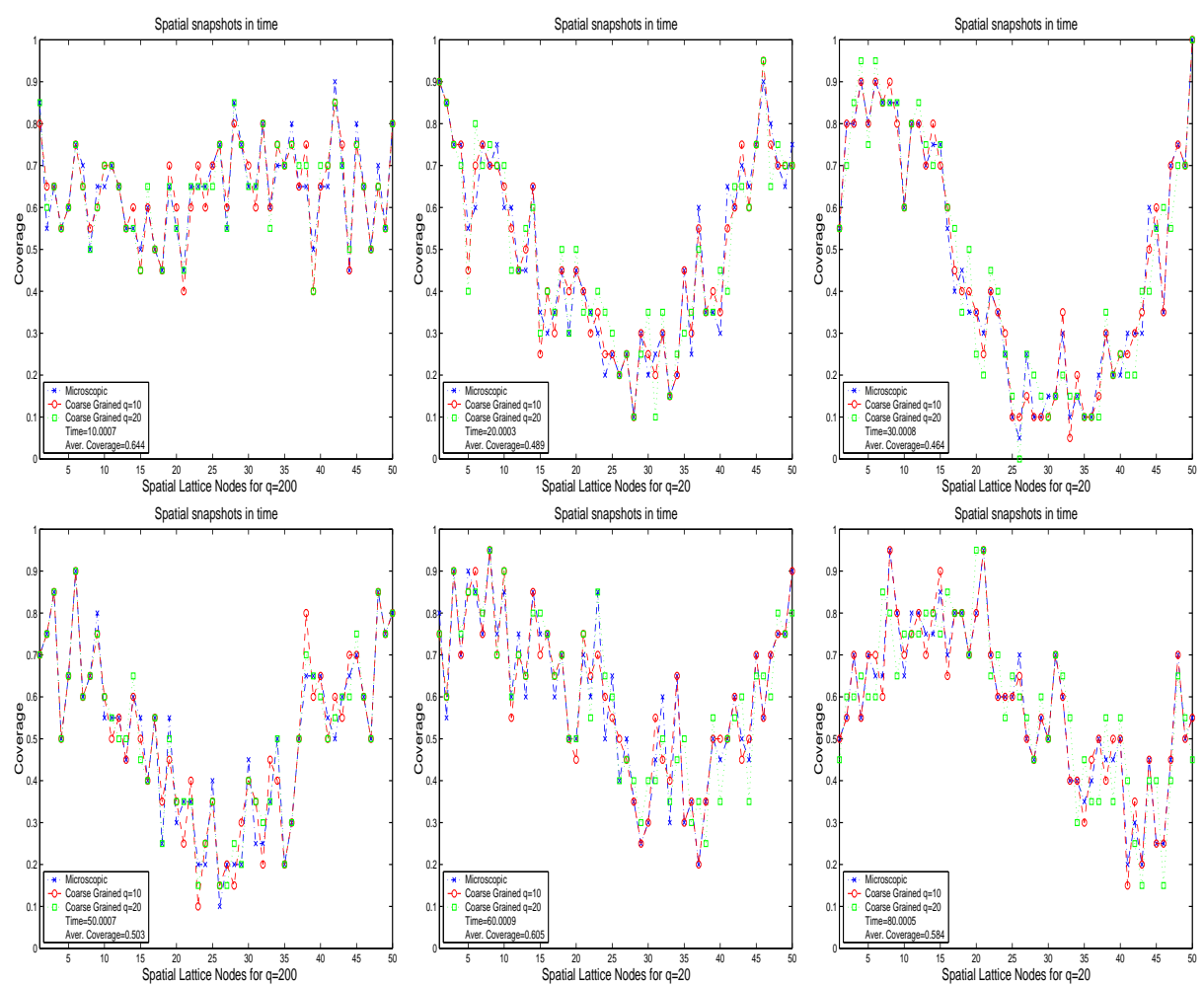

Figure 19. Oscillatory example for $L=200$. Time series for $\eta$ between microscopic $q=1$ and $q=10,20$. This a phase transition regime since $\beta J_{0}=6$. Other parameters: $c_{a}=c_{d}=1, a=100, b=$ $0, c=1, \alpha=.01, \gamma=0$ and $N=1000$.

and coarse grained dynamics is more pronounced. We observe disagreement in both spatial and temporal variables of interest between $q=1$ and any of the other coarse graining examined. Overall however, as we have seen before, there is agreement in means.

This is a challenging case and the statistical signal analysis will allow us to see sometimes hidden trends over long times. Overall we observe good agreement for the case of $q=10$ and $q=1$ as can be seen in [KMSa] as can be seen by the periodograms included there. This is expected since according to our Theorem 7.1 $q$ is small enough compared to $L$ and the approximation does not produce much error.

Remarkably the cases of $q=1$ and $q=50$ as presented in the ensemble periodograms in Figures 23 and 24 also display good agreement although you might have thought otherwise by simply comparing the raw signal included in these figures.

We also provide $l_{1}$ error estimates in Table 8.2.2 for the differences observed in the ensemble power spectrum for both of the examples above (we only present the case with $L=200$ for the oscillatory case). 

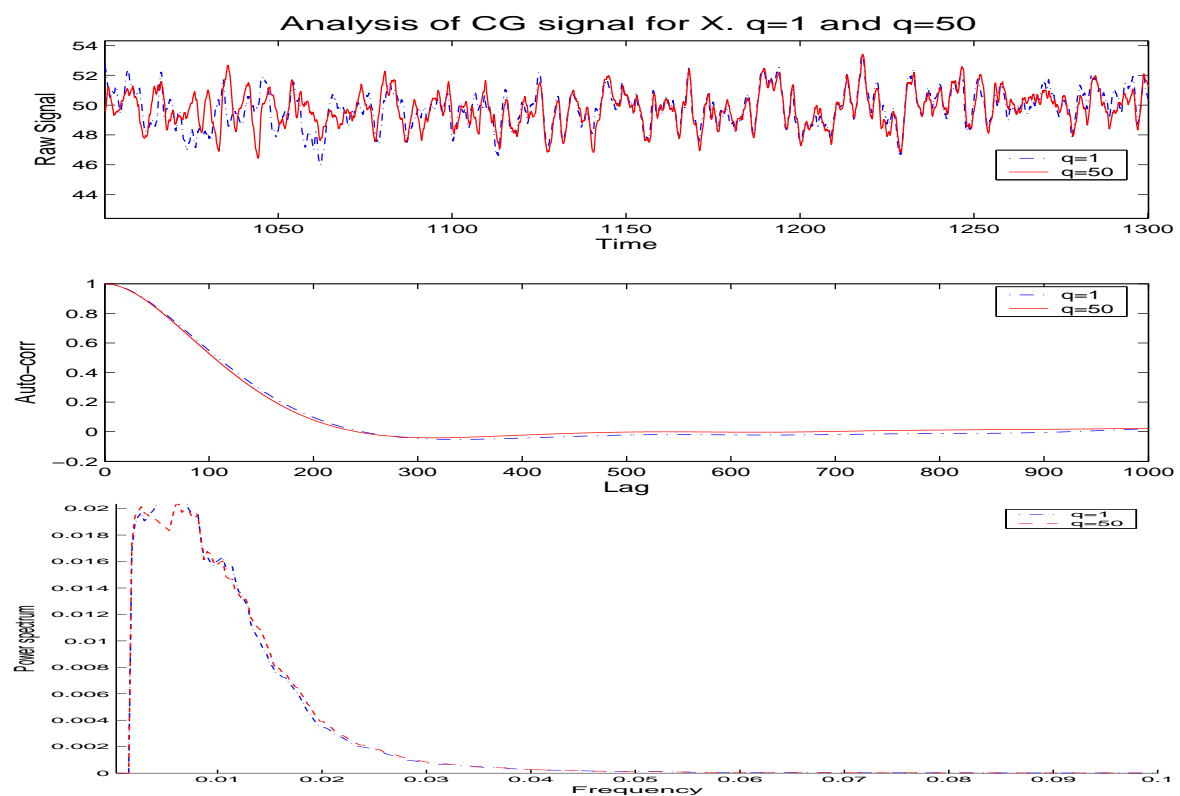

Figure 20. Comparisons for oscillatory case on signal from $X$ with $L=100$. Timeseries, auto-correlation and spectrum analysis for the cases of $q=1$ and $q=50$ with $\beta J_{0}=6$.

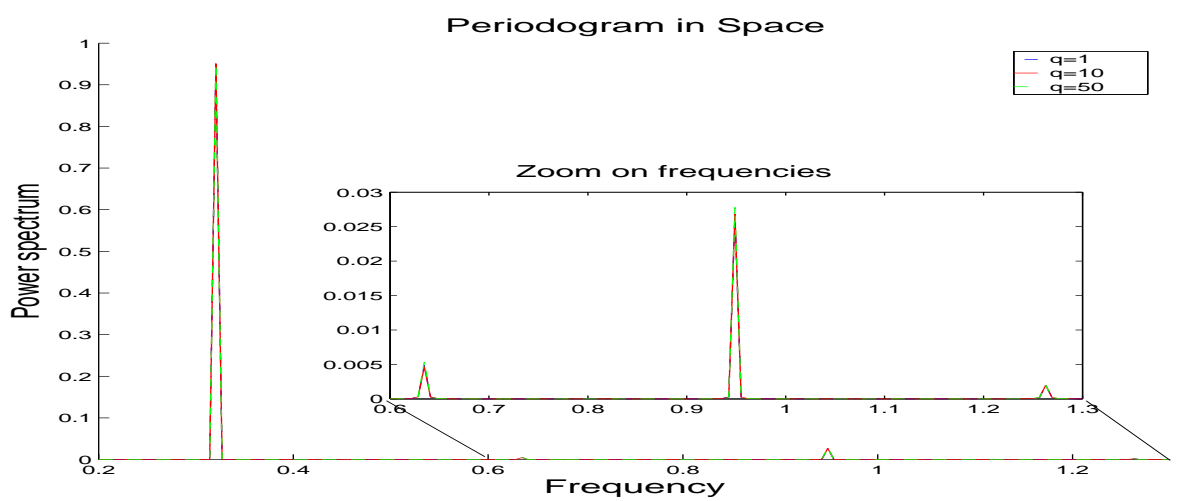

FIgURE 21. Spatial comparisons for $X$ in the oscillatory case with $L=100$. Spatial spectrum analysis for the cases of $q=1,10$ and 50. For this comparison spatial domains are adjusted to that corresponding to $q=50$ (i.e. $m=20$, where $N=m q$ ) with $\beta J_{0}=6$.

Similarly we note that once again we have significant CPU time improvement. We provide the CPU times for the excitable and oscillatory examples in Tables 8.3 and 8.4 respectively. The microscopic hybrid system corresponds to $q=1$ as usual. We observe in the tables below that each time we double the coarse graining parameter $q$ we improve the CPU time by more than double. We also note that the excitable case seems to have taken far more CPU time than the oscillatory did. 

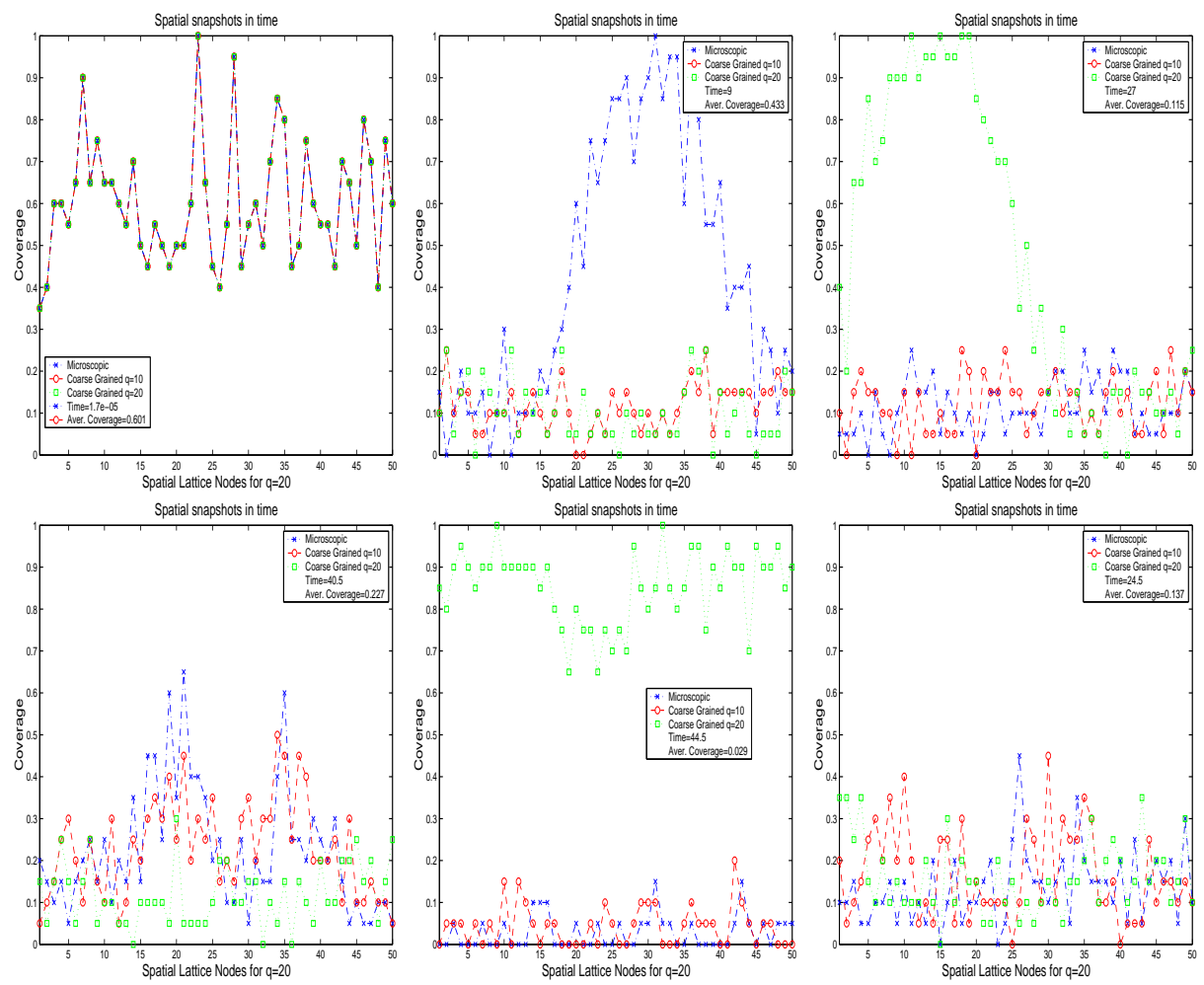

Figure 22. Excitable case. Snapshots in time for $\eta$ between microscopic $q=1$ and $q=10,20$ under the phase transition $\beta J_{0}=6$. Single stable node exhibiting pulses and random departures. Other parameters: $c_{a}=c_{d}=100, a=100, b=30, c=1, \alpha=.01, \gamma=0$ with $L=100$ and $N=1000$.

\begin{tabular}{|c|c|c|}
\hline Spectral error & $\left\|G^{q_{1}}-G^{q_{10}}\right\|_{1}$ & $\left\|G^{q_{1}}-G^{q_{50}}\right\|_{1}$ \\
\hline$G \equiv X_{o s c}$ & $\begin{array}{l}p=0: .7 \\
p=4: .3\end{array}$ & $\begin{array}{l}p=0: 1.1 \\
p=4: .5\end{array}$ \\
\hline$G \equiv u_{o s c}$ & $\begin{array}{l}p=0: .8 \\
p=4: .3\end{array}$ & $\begin{array}{l}p=0: 1.3 \\
p=4: .5\end{array}$ \\
\hline$G \equiv X_{e x c}$ & $\begin{array}{l}p=0: .9 \\
p=4: .3\end{array}$ & $\begin{array}{l}p=0: 1.5 \\
p=4: .7\end{array}$ \\
\hline$G \equiv u_{e x c}$ & $\begin{array}{l}p=0: 1.1 \\
p=4: .3\end{array}$ & $\begin{array}{l}p=0: 1.6 \\
p=4: .5\end{array}$ \\
\hline
\end{tabular}

TABLE 8.2. The $l_{1}$ error estimate between microscopic and coarse grained solutions for the oscillatory $(L=200)$ and excitable $(L=$ 100 ) examples in this section. Here $p$ refers to the post-averaging local smoothing used. For details on smoothing techniques we refer to the Appendix of [KMSa]

This is due to the fact that our algorithm is adaptive and therefore chooses smaller step sizes for the excitable case. 


\begin{tabular}{|c||c|c|c|c|}
\hline $\mathrm{q}$ & 1 & 10 & 20 & 50 \\
\hline \hline CPU Time (sec) & 2412131 & 27122 & 9150 & 2281 \\
\hline
\end{tabular}

TABle 8.3. Excitable case. CPU times for solutions of the microscopic hybrid system and the corresponding coarse grained closure. Parameters: $L=100, N=1000$.

For $L=200$ :

For $L=20$ :

\begin{tabular}{|c||c|c|c|c|c|c|}
\hline q & 1 & 10 & 20 & 40 & 50 & 100 \\
\hline \hline CPU Time (sec) & 19520 & 407 & 108 & 33 & 22 & 7 \\
\hline q & 1 & 10 & 20 & & & \\
\hline CPU Time (sec) & 213 & 55 & 18 & & & \\
\end{tabular}

TABLE 8.4. Oscillatory case. CPU times for solutions of the microscopic hybrid system against the corresponding coarse grained closure. Parameters: $N=1000$.

Overall we note that the coarse grained strategy has been systematically tested in rather demanding regimes and performs well in terms of error of approximation. Further the CPU savings, which were reported earlier in Section 5.1 and here in Tables 8.3 and 8.4 are substantial.
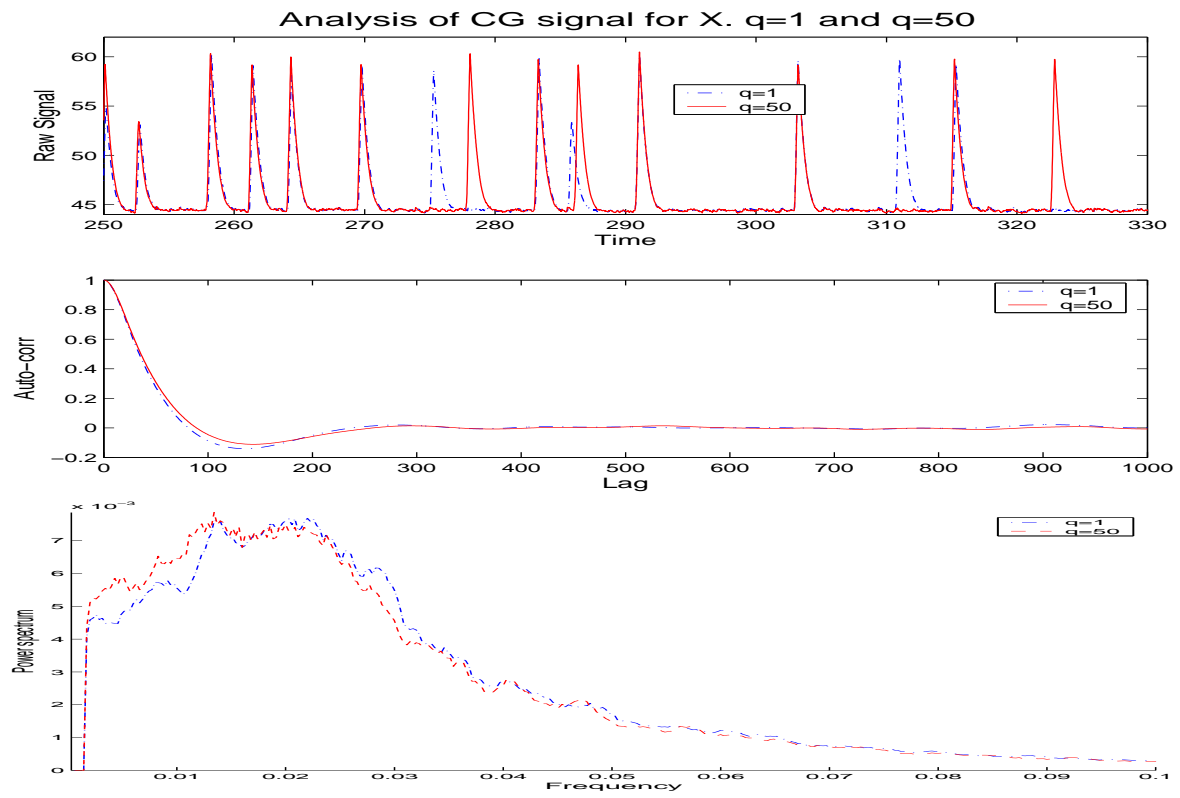

Figure 23. Comparisons for excitable case on signal from $X$. Timeseries, auto-correlation and spectrum analysis for the cases of $q=1$ and 50. Parameters: $L=100, N=1000$ and $\beta J_{0}=6$. 

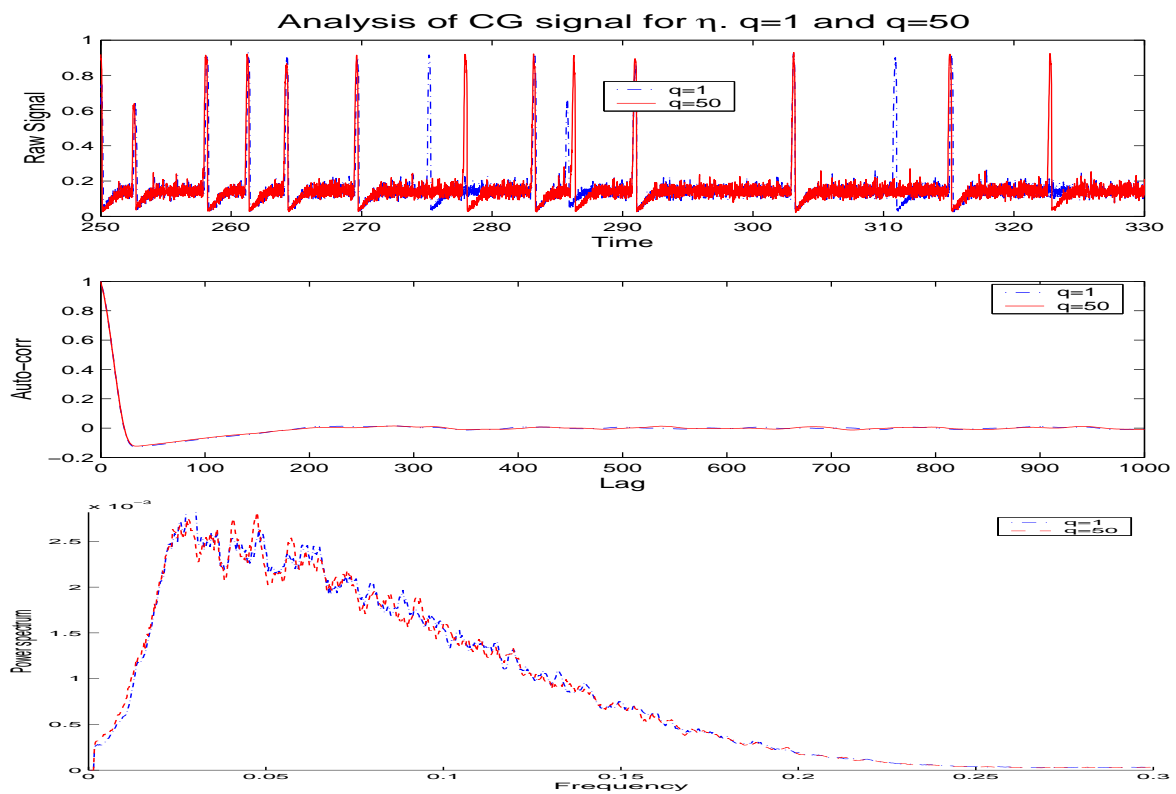

Figure 24. Comparisons for excitable case on signal from $\eta$. Timeseries, auto-correlation and spectrum analysis for the cases of $q=1$ and 50. Parameters: $L=100, N=1000$ and $\beta J_{0}=6$.

\section{Conclusions}

In this paper we studied the influence of micro- or sub- grid scale noise in hybrid dynamical systems consisting of coupled macroscopic, deterministic differential equations and microscopic, stochastic lattice particle systems. In particular we developed mathematical prototype hybrid models coupling stochastic and deterministic systems and derived deterministic mesoscopic models in various asymptotic limits in order to evaluated the extent of validity of theoretical predictions by extensive direct numerical simulations and comparisons. We have undertaken our study for systems with or without short microscopic interactions in the lattice system. In either case our findings suggest that deterministic closures of the hybrid system such as mean field, stochastic averaging or moment equations may be either inadequate as descriptions of the overall system or simply difficult to both derive and assess their effectiveness. Specifically, we have established that deterministic closures have substantial deviations from the hybrid system due to the microscopic fluctuations. This is shown in both transient regimes with high noise but also for the case of weak noise in long-time regimes, where rare events may become significant.

In that respect we subsequently developed systematic derivations of stochastic mesoscopic models by employing Coarse Grained Monte Carlo (CGMC) models. Implementation of these CGMC systems reveals that not only we obtain the solutions which govern the hybrid system in mean field but also reproduce the stochastic noise which as we have seen here is essential, at least in regimes where the CGMC approximation is valid. Furthermore the success of the method is also validated for the phase transition regime where metastable, intermittent and oscillatory phenomena take effect thus validating the CGMC closure presented for an even wider 
context encompassing complex stochastic dynamics. In particular, based on the simulations our findings for the coarse grained closure are as follows: (a) Agreement with microscopic dynamics even if there is no scale separation between the deterministic and stochastic models (b) Pathwise agreement (c) Agreement even in case of solutions with stable nodes which switch in time due to fluctuations. Those are solutions which due to the inherent stochastic noise in the system move between separate stable states; the coarse grained simulation follows these moves exactly for very long times (d) Remarkable CPU time improvement based on the size $q$ of coarse graining used. (e) Care must be taken so as to not over-coarse grain since in that case we see a deterioration in accuracy of the coarse grained simulation. There is a relation between the size of the potential radius $L$ and the size of coarse graining $q$ after which this deterioration starts to occur, which is mathematically quantified by the relative entropy estmates in Section 7.1. Similarly, based on Theorem 7.1, the nearest neighbor case of $L=1$ should produce higher approximation errors. Cases with smaller interaction potentials $(L=10)$ have also been examined successfully in [KPS05] for uncoupled dynamics at least in the presence of a strong external field but the extend for which the approximation will succeed for small $L$ should be probed further for hybrid systems.

Stochastic closures for hybrid systems with strong interactions (corresponding to phase transitions in the lattice model) focused on examples dominated by strong interactions between scales and fluctuations such as nucleation, intermittent and random oscillations regimes. This was further demonstrated in terms of detailed comparisons to direct numerical simulations. The coarse-grained stochastic closures yield statistical agreement with the full hybrid system. This result was substantiated with time series analysis for the original system and its stochastic approximations that included comparisons of power spectra, auto- and cross- correlations in time and space. We refer to $[\mathbf{K M S a}]$ for more details and examples.

\section{References}

[AK02] R. S. Aranson and L. Kramer, The world of the complex Ginzburg-Landau equation, Reviews of Modern Physics 74 (2002), 99-143.

[BSL60] R. B. Bird, W. E. Stewart, and E. N. Lightfoot, Transport phenomena, Wiley, 1960.

[EE03] W. E and B. Engquist, The heterogeneous multiscale method, Comm. Math. Sci. (2003), 87-133.

[Ell85] R. Ellis, Entropy, large deviations, and statistical mechanics, Springer-Verlag, 1985.

[FW98] M. I. Friedlin and A. D. Wentzell, Random perturbations of dynamical systems, Springer, 1998.

[GHJM98] L. Grammaitoni, P. Hanggi, P. Jung, and F. Mardesoni, Stochastic resonance, Reviews of Modern Physics 70 (1998), 223-287.

$\left[\mathrm{KGH}^{+} 03\right]$ I. G. Kevrekidis, C. W. Gear, J. Hyman, P. G. Kevrekidis, O. Runborg, and C. Theodoropoulos, Equation-free, coarse-grained multiscale computation: enabling microscopic simulators to perform system-level tasks, Comm. Math. Sci. (2003), 715762.

[KL99] C. Kipnis and C. Landim, Scaling limits of interacting particle systems, Springer, 1999.

[KMSa] M. A. Katsoulakis, A. J. Majda, and A. Sopasakis, Intermittency, metastability and coarse graining for coupled deterministic-stochastic systems, submitted.

[KMSb] Multiscale couplings in prototype hybrid deterministic/stochastic systems: Part II, stochastic closures, Communications in Mathematical Sciences (2005), to appear. 
[KMS04] - Multiscale couplings in prototype hybrid deterministic/stochastic systems: Part I, deterministic closures, Communications in Mathematical Sciences (2004), 255294.

[KMV03a] M. A. Katsoulakis, A. J. Majda, and D. G. Vlachos, Coarse-grained stochastic processes and monte carlo simulations in lattice systems, J. Comp. Phys. 186 (2003), $250-278$.

[KMV03b] _ Coarse-grained stochastic processes for lattice systems, Proc. Natl. Acad. Sci. 100 (2003), 782-787.

[KPS05] M. A. Katsoulakis, P. Plecháč, and A. Sopasakis, Error control and analysis in coarsegraining of stochastic lattice dynamics, submitted.

[KSA84] I. G. Kevrekidis, L. D. Schmidt, and R. Aris, Surf. Sci. 137 (1984), 151.

[KT06] M. A. Katsoulakis and J. Trashorras, Information loss in coarse-graining of stochastic particle dynamics, J. Stat. Phys. (2006), in press.

[KV03] M. A. Katsoulakis and D. G. Vlachos, Hierarchical kinetic Monte Carlo simulations for diffusion of interacting molecules, J. Chem. Phys. 112 (2003), no. 18.

[LB99] D. A. Lavis and G. M. Bell, Statistical mechanics of lattice systems 1: Closed-form and exact solutions, Springer, 1999.

[Lig99] T. M. Liggett, Stochastic interacting systems: Contact process, Voter and exclusion process, Springer-Verlag, Berlin Heidelberg, 1999.

[MK02] A. J. Majda and B. Khouider, Stochastic and mesoscopic models for tropical convection, Proc. Nat. Acad. Sci. 99 (2002), 1123

[MTE01] A. J. Majda, I. Timofeyev, and E. Vanden Eijnden, A mathematical framework for stochastic climate models, Comm. Pure Appl. Math. 54 (2001), 891.

[Mur89] J. D. Murray, Mathematical biology, Springer-Verlag, 1989.

[OL04] H. C. Oettinger and M. Laso, Simulating polymer flow, Simulation Methods for Modeling Polymers (M. J. Kotelyanski and D. N. Theodorou, eds.), 2004.

[RJ98] S. T. Rodgers and K. F. Jensen, Multiscale modeling of chemical vapor deposition, J. Appl. Phys. 83 (1998), no. 1, 524.

[CT91] J. A. Cover and T. M. Thomas, Elements of information theory, Wiley, 1991.

[VSA90] D. G. Vlachos, L. D. Schmidt, and R. Aris, The effects of phase transitions, surface diffusion and defects on surface catalyzed reactions: Fluctuations and oscillations, J. Chem. Phys. 93 (1990), 8306.

[WSL03] H. S. Wiley, S. Y. Shvartsman, and D. A. Lauffenburger, Computational modeling of the egf receptor system: a paradigm for systems biology, Trends Cell Biol. 13 (2003), $43-50$.

[ZGB86] R. M. Ziff, E. Gulari, and Y. Barshad, Kinetic phase transitions in an irreversible surface-reaction model, Phys. Rev. Lett. 56 (1986), 2553.

Department of Mathematics and Statistics, University of Massachusetts, Amherst, MA 01003-9305

E-mail address: markos@math.umass.edu

Courant Institute of Mathematical Sciences and Center for Atmospheric and Ocean Sciences, New York University, New York, NY 10012

E-mail address: jonjon@math.cims.nyu.edu

Department of Mathematics and Statistics, University of Massachusetts, Amherst, MA 01003-9305

E-mail address: sopas@math.umass.edu 